\title{
Holographic drag force in 5d Kerr-AdS black hole
}

\author{
Irina Ya. Aref'eva, ${ }^{a}$ Anastasia A. Golubtsova ${ }^{b, c}$ and Eric Gourgoulhon ${ }^{d}$ \\ ${ }^{a}$ Steklov Mathematical Institute, Russian Academy of Sciences, \\ Gubkina str. 8, 119991, Moscow, Russia \\ ${ }^{b}$ Bogoliubov Laboratory of Theoretical Physics, Joint Institute for Nuclear Research, \\ Joliot-Curie str. 6, Dubna, 141980 Russia \\ ${ }^{c}$ Dubna State University, \\ Universitetskaya str. 19, Dubna, 141980, Russia \\ ${ }^{d}$ Laboratoire Univers et Théories, Observatoire de Paris, CNRS, \\ Université PSL, Université de Paris, \\ 5 place Jules Janssen, 92190 Meudon, France \\ E-mail: arefeva@mi-ras.ru, golubtsova@theor.jinr.ru, \\ eric.gourgoulhon@obspm.fr
}

ABSTRACT: We consider the 5d Kerr-AdS black hole as a gravity dual to rotating quarkgluon plasma. In the holographic prescription we calculate the drag force acting on a heavy quark. According to the holographic approach a heavy quark can be considered through the string in the gravity dual. We study the dynamics of the string for the Kerr-AdS backgrounds with one non-zero rotational parameter and two non-zero rotational parameters that are equal in magnitude. For the case of one non-zero rotational parameter we find good agreement with the prediction from the $4 \mathrm{~d}$ case considered by arXiv:1012.3800.

KEYworDs: Holography and quark-gluon plasmas, AdS-CFT Correspondence, Bosonic Strings

ARXIV EPRINT: 2004.12984 


\section{Contents}

1 Intoduction 1

2 Setup $\mathbf{3}$

2.1 Drag forces in QGP as resistance to string moving in holographic space 3

2.2 Drag forces via hydrodynamic equations 5

$\begin{array}{lll}2.3 & \text { Drag forces and special Wilson loops } & 7\end{array}$

$2.4 D=5$ Kerr-AdS black holes 8

$\begin{array}{ll}2.5 & \text { Thermodynamics of the 5d Kerr-AdS black holes } \\ & 12\end{array}$

3 Drag force in 5d Kerr-AdS background with one rotational parameter $\mathbf{1 5}$

$\begin{array}{lll}3.1 & \text { Static curved string in the 5d Kerr-AdS background } & 15\end{array}$

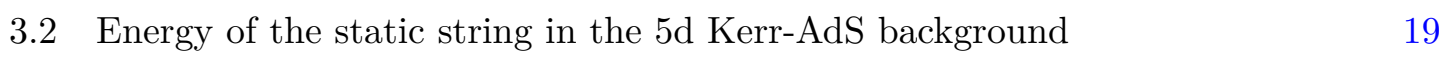

4 Drag force from the 5d Kerr-AdS metric with two equal rotational parameters $\quad 22$

4.1 Static curved string in the Boyer-Lindquist coordinates 22

4.2 Static curved string in global AdS coordinates 24

5 Conclusion and discussion $\quad 26$

A Some facts on Kerr-AdS black holes $\quad 29$

A.1 Special cases of 5d Kerr-AdS black holes 29

$\begin{array}{lll}\text { A.2 Boundaries } & 29\end{array}$

B Strings in a 5d Kerr-AdS black hole $\quad 30$

B.1 Straight string solution in global AdS 30

B.2 Curved string in 5d Kerr-AdS with one rotational parameter (supplementary relations)

\section{Intoduction}

Starting with Landau's works [1], the hydrodynamic description of the quark-gluon plasma (QGP) is the main theoretical method for linking the QGP models with experimental results. Experimental data, as a rule, are distributions by energy, momenta, spin polarization, and types of particles, etc. Parameters of the QGP fluid are primary important and are fitted to describe experimental data. After several tests it is believed that the QGP fluid satisfies the relativistic ideal hydrodynamics equations, in the simplest case such as the Bjorken [2] or Gubser's ones [3]. Vorticity is an important characteristic of fluids [4-8]. Recent experiments by the STAR collaboration at the RHIC facility [9-12] indicate that 
the QGP fluid produced in peripheral relativistic heavy-ion collisions is in fact a rotating fluid. It was proposed to use the average polarization of $\Lambda$ hyperons to experimentally estimate the fluid vorticity in heavy-ion collisions. The global spin polarization of $\Lambda / \bar{\Lambda}$ hyperons related with vorticity was observed at RHIC [9-12] (see [13-15] for more details). The angular momentum $J$ is shown to be in the range of $10^{3} \hbar-10^{5} \hbar[6,7]$. Rotation is also important for neutron starts [16].

On the other hand, a powerful method for the theoretical study of QGP is the holographic approach, which is closely related to the hydrodynamic method [17-19]. As was established by Bhattacharyya et al. [20,21], the rotating fluid models are associated with rotating AdS (Kerr-AdS) black holes. A description of dynamics of fluid flows through the conformal Navier Stokes equations with transport coefficients holographically were computed in the 5d Kerr-AdS background and the dual field stress-energy tensor was calculated in $[20,21]$. In the paper [22] a solution of relativistic ideal hydrodynamics that describes rotation around two axes and parametrized by two parameters, the first one is related with non-vanishing angular momentum for off-center collisions, while the second one parametrizes inhomogeneities of incoming nuclei, is constructed and its relevance to experimental data is shown. Recently, the effects of rotation on the hydrodynamic quasinormal modes of spinning black hole were studied in [23].

The holographic duality relates the properties of black holes in an AdS spacetime to the thermal properties of dual conformal field theories at strong coupling in a spacetime of smaller dimension. The five dimensional rotating black holes with AdS asymptotics were discovered in [24]. 5d Kerr-AdS metrics due to $\mathrm{SO}(4)$-symmetry are characterized by two rotation parameters that are associated with the number of Casimirs for $S O(4)$ and are preserved independently. Kerr-AdS black holes have been studied in the framework of holographic duality [24]. In this case CFT duals to Kerr-AdS black holes are CFTs in a 4d rotating Einstein universe. ${ }^{1}$ Application of Kerr AdS/CFT duality to QGP is justified at hight energies reached at LHC and RHIC, since in these regions the restoration of conformal invariance takes place. Note, that in dual conformal theories operators are characterized by two quantum numbers, dimension and spin, the last of which has two independent parts if the theory is defined on $\mathbb{R} \times \mathbb{S}^{3}$.

Within holographic applications Kerr-AdS black holes were discussed for both highenergy physics and condensed matter phenomena [20]-[37].

One of the well-tested holographic predictions are related with energy loss calculations [38]-[46], in particular, jet suppression calculations [40]. To model QGP phase transition these calculations were made for non-conformal isotropic [47] and anisotropic [48-50] backgrounds, some of them taking into account the motion of the QGP fluid [51, 52]. In context of recent interest to a rotational vorticity and the efforts to get this quantity experimentally it is of interest to perform a holographic calculation of energy loss in a rotating fluid. This is the main goal of the paper.

\footnotetext{
${ }^{1}$ After [24], a number of interesting facts concerning the Kerr-AdS/CFT correspondence were found out $[25]-[29]$.
} 
For our analysis we follow-up the holographic prescription [38, 39] where moving of a heavy quark in QGP is dual to an open string endpoint at the boundary of the background under consideration. In our case the string is stretched down from the Kerr-AdS space boundary to the black hole horizon. The dynamics of the string is described by the NambuGoto action. To solve the corresponding equations we generalize an approach proposed for more simple Kerr- $A d S_{4}$ case of [32]. This approach consists in expanding an ansatz for solutions to string equations of motion in series by a rotating parameter. We write string equations of motion in Kerr- $A d S_{5}$, expand them on rotations parameters and solve the linearized version of these equations of motion. Then we find the components of conjugate momenta. We perform calculations both in Boyer-Lindquist (rotating-at-infinity frame) and AdS (static-at-infinity frame) coordinate systems. In this paper we consider explicitly two cases: a) rotational parameters are non-zero and equal in the absolute value; b) one of rotational parameters vanishes. The third case will be the subject of separated considerations. For the extremal black holes (with merging horizons) that are related to the case with equal rotational parameters we observe vanishing of the drag force.

The paper is organized as follows. Section 2 consist of several sections that contain the description of main tools using in this paper. In section 2.1 we briefly remind the main steps of the drag force calculations. In section 2.2 we remind how the drag forces appear hydrodynamic equations. In section 2.3 we present the relation between drag forces and special Wilson loops, and notice the difference of this connection between a spherical symmetric case considered here and a plane AdS case. In section 2.4 we describe thermodynamics of the 5d Kerr-AdS black holes. In section 3 we find the drag force for a fixed quark studying string dynamics in a 5d Kerr-AdS black hole with one rotational parameter. In section 3.1 we find solutions to linearized string equations of motion. Assuming that the rotational parameter is small we find the conjugate momenta and the leading term for the drag force. Then in section 3.2 we calculate corrections to the thermal quark mass at rest. In section 4 we discuss the case with two rotational parameters that are equal in magnitude. We perform computations of the drag force in Boyer-Lindquist and global AdS coordinates in sections 4.1 and 4.2 , respectively. In section 5 we conclude with a summary of the presented results as well with outline of future directions mainly related with NICA that requires the calculations in the deformed Kerr-AdS metric. In the appendix, we keep supplementary relations, that are useful for the main calculations.

\section{Setup}

\subsection{Drag forces in QGP as resistance to string moving in holographic space}

The drag force is a force acting opposite to the relative motion of the heavy quark moving with respect to a surrounding quark-gluon plasma. Drag forces define the energy loss in QGP. This is an important property and there is a large literature dedicated to this subject [38]-[46] and refs. therein. Following the dictionary of the gauge/gravity duality the heavy quark is associated to an endpoint of a relativistic string suspended from the boundary of the Kerr-AdS background into the interior [38, 39]. Here we briefly describe this 
approach for an arbitrary curved background. In the holographic approach to investigate quark dynamics one has to study the string motion described by the Nambu-Goto action

$$
S_{N G}=-\frac{1}{2 \pi \alpha^{\prime}} \int d \sigma^{0} d \sigma^{1} \sqrt{|g|},
$$

where $g=\operatorname{det} g_{\alpha \beta}$ is the determinant of the induced metric which is defined though the $5 \mathrm{~d}$ spacetime metric $G_{\mu \nu}$ (in section3 and 4 this will corresponds to the 5d Kerr-AdS black hole metric) by

$$
g_{\alpha \beta}=G_{\mu \nu} \partial_{\alpha} X^{\mu} \partial_{\beta} X^{\nu},
$$

$X^{\mu}$ are the embedding functions of the string worldheet in the spacetime, we also assume that $X^{\mu}=X^{\mu}(\sigma)$. The equations of motions have the form

$$
\frac{1}{\sqrt{-g}} \partial_{\alpha}\left(\sqrt{-g} G_{\mu \nu} \partial^{\alpha} X^{\nu}\right)-\frac{1}{2} \partial_{\mu} G_{\rho \nu} \partial_{\alpha} X^{\rho} \partial^{\alpha} X^{\nu}=0 .
$$

The conserved currents are defined as variational derivatives on $\partial_{\alpha} X^{\mu}$

$$
\pi_{\mu}^{\alpha} \equiv-2 \pi \alpha^{\prime} \frac{\delta S}{\delta \partial_{\alpha} X^{\mu}}=\sqrt{-g} g^{\alpha \beta} G_{\mu \nu}(X) \partial_{\beta} X^{\nu} .
$$

Note, that this current is related to the translational invariance.

In (2.1)-(2.2) we define $\sigma^{\alpha}$ with $\alpha=0,1$ as the string worldsheet coordinates. So for the conjugated momenta $\pi_{\mu}^{\alpha}$ one can write

$$
\partial_{\alpha} \pi_{\mu}^{\alpha}=0, \quad \partial_{\alpha} \pi_{\mu}^{\alpha}=\frac{\partial \pi_{\mu}^{\sigma^{0}}}{\partial \sigma^{0}}+\frac{\partial \pi_{\mu}^{\sigma^{1}}}{\partial \sigma^{1}} .
$$

The corresponding charge reads

$$
\begin{aligned}
\int_{M} \partial_{\alpha} \pi_{\mu}^{\alpha} d \sigma^{2} & =\int_{M}\left(\frac{\partial \pi_{\mu}^{\sigma^{0}}}{\partial \sigma^{0}}+\frac{\partial \pi_{\mu}^{\sigma^{1}}}{\partial \sigma^{1}}\right) d \sigma^{0} d \sigma^{1} \\
& =\int_{\partial_{M}}-\pi_{\mu}^{\sigma^{1}} d \sigma^{0}+\int_{\partial_{M}} \pi_{\mu}^{\sigma^{0}} d \sigma^{1}=\int_{\partial M} \vec{\pi}_{\mu} d \vec{\sigma}
\end{aligned}
$$

where $M$ is a two-dimensional worldsheet manifold and $\partial M$ is its boundary. The associated conserved charge is the total momentum in the $\mu$-direction

$$
p_{\mu}=\int d \Sigma_{\alpha} \pi_{\mu}^{\alpha}
$$

where $\Sigma_{\alpha}$ is a cross-sectional surface on the worldsheet. Taking into account $\Sigma_{\sigma^{1}}=$ $d \sigma^{0} \sqrt{-g_{00}} \hat{n}_{\sigma^{1}}$ with

$$
\hat{n}^{\alpha}=\left(-\frac{g_{10}}{\sqrt{-g g_{11}}}, \sqrt{\frac{g_{11}}{-g}}\right),
$$

the time-independent force on the string is

$$
\frac{\partial p_{\mu}}{\partial \sigma^{0}}=-\frac{1}{2 \pi \alpha^{\prime}} \pi_{\mu}^{\sigma^{1}}
$$


Therefore, to find the components of the drag force (2.9) we need to calculate the conjugate momenta of the string (2.4).

The total energy of the string is given by

$$
E=-\frac{1}{2 \pi \alpha^{\prime}} \int d r \pi_{t}^{\sigma^{0}}
$$

where the conjugate momentum $\pi_{t}^{\sigma^{0}}$ is calculated using (2.4). In what follows we simplify notations: $\pi_{t}^{\sigma^{0}}=\pi_{t}^{0}, \pi_{t}^{\sigma^{1}}=\pi_{t}^{r}$.

Drag forces in the holographic approach have been calculated for several isotropic models including models describing the quark confinement/deconfinement and chiral symmetry breaking phase transitions [38, 39, 41, 46, 52, 53]. Drag forces in anisotropic QGP using the suspected string are calculated in [48-50,54-57] including plasma with magnetic field [58-62].

\subsection{Drag forces via hydrodynamic equations}

Here we show that the drag force can be considered as a relativistic pressure gradient force in the fluid. Originally this was demonstrated in [32] for the $3 \mathrm{~d}$ case. Here we consider a rotating fluid in a 4-dimensional spacetime $\mathbb{R} \times \mathbb{S}^{3}$ that has the metric

$$
d s^{2}=-d T^{2}+\frac{1}{\ell^{2}}\left(d \Theta^{2}+\sin ^{2} \Theta d \Phi^{2}+\cos ^{2} \Theta d \Psi^{2}\right) .
$$

The metric has the following non-zero Christoffel symbols

$$
\Gamma_{33}^{2}=-\frac{1}{2} \sin (2 \Theta), \quad \Gamma_{44}^{2}=\frac{1}{2} \sin (2 \Theta), \quad \Gamma_{32}^{3}=\cot \Theta, \quad \Gamma_{42}^{4}=-\tan \Theta
$$

The stress-energy tensor in hydrostationary equilibrium reads

$$
T^{A B}=(\rho+P) u^{A} u^{B}+P g^{A B},
$$

where $g^{A B}$ are components of the metric (2.11), $A, B=1, \ldots, 4, \rho$ is the density, $P$ is the pressure, and $u^{A}$ is the velocity field:

$$
u^{A}=\frac{1}{\sqrt{1-\ell^{-2} \Omega_{\phi}^{2} \sin ^{2} \Theta-\ell^{-2} \Omega_{\psi}^{2} \cos ^{2} \Theta}}\left(1,0, \Omega_{\phi}, \Omega_{\psi}\right),
$$

so $u^{A} u_{A}=-1, \Omega_{\phi}=a \ell^{2}, \Omega_{\psi}=b \ell^{2}$.

Correspondingly, taking into account $u_{A}=g_{A B} u^{B}$ we also have

$$
u_{A}=\frac{1}{\sqrt{1-\ell^{-2} \Omega_{\phi}^{2} \sin ^{2} \Theta-\ell^{-2} \Omega_{\psi}^{2} \cos ^{2} \Theta}}\left(-1,0, \ell^{-2} \Omega_{\phi} \sin ^{2} \Theta, \ell^{-2} \Omega_{\psi} \cos ^{2} \Theta\right) .
$$


Let's put $\ell^{-2} \Omega_{\Phi}^{2} \sin ^{2} \Theta+\ell^{-2} \Omega_{\Psi}^{2} \cos ^{2} \Theta=v^{2},\left(1-v^{2}\right)^{-1 / 2}=\gamma$ and following [20] we get

$$
T^{A B}=\gamma^{2}\left(\begin{array}{cccc}
\rho+P v^{2} & 0 & (\rho+P) \Omega_{\phi} & (\rho+P) \Omega_{\psi} \\
0 & P \ell^{2} \gamma^{-2} & 0 & 0 \\
(\rho+P) \Omega_{\phi} & 0 & \rho \Omega_{\phi}^{2}+P\left(\ell^{2} \csc ^{2} \Theta-\Omega_{\psi}^{2} \cot ^{2} \Theta\right) & (\rho+P) \Omega_{\phi} \Omega_{\psi} \\
(\rho+P) \Omega_{\psi} & 0 & (\rho+P) \Omega_{\phi} \Omega_{\psi} & \rho \Omega_{\psi}^{2}+P\left(\ell^{2} \sec ^{2} \Theta-\Omega_{\phi}^{2} \tan ^{2} \Theta\right)
\end{array}\right)
$$

the same as eq. (30) [20]. Taking $\rho=3 P$ and $3 P=3 \tau^{4} \gamma^{4}$ we obtain

$$
T^{A B}=\gamma^{6} \tau^{4}\left(\begin{array}{cccc}
\left(3+v^{2}\right) & 0 & 4 \Omega_{\phi} & 4 \Omega_{\psi} \\
0 & \ell^{2}\left(1-v^{2}\right) & 0 & 0 \\
4 \Omega_{\phi} & 0 & 3 \Omega_{\phi}^{2}+\ell^{2} \csc ^{2} \Theta-\Omega_{\psi}^{2} \cot ^{2} \Theta & 4 \Omega_{\phi} \Omega_{\psi} \\
4 \Omega_{\psi} & 0 & 4 \Omega_{\phi} \Omega_{\psi} & 3 \Omega_{\psi}^{2}+\ell^{2} \sec ^{2} \Theta-\Omega_{\phi}^{2} \tan ^{2} \Theta
\end{array}\right)
$$

that coincides with (eq.34) [20] and (eq.11) [22].

The corresponding conservation law of the stress-energy tensor is

$$
\nabla_{A} T^{A B}=0
$$

One can rewrite the conservation law projecting (2.18) onto the direction orthogonal to the velocity field

$$
\left(g_{B C}+u_{B} u_{C}\right) \nabla_{A} T^{A C}=0,
$$

that can be rewritten as

$$
\left(g_{B C}+u_{B} u_{C}\right) \nabla^{C} P+(\rho+P) u^{C} \nabla_{C} u_{B}=0 .
$$

Owing to rotational symmetry we have the dependence only on $\Theta$ for $\rho$ and $P$ :

$$
\rho=\rho(\Theta), \quad P=P(\Theta),
$$

that reduces to the following equation

$$
\partial_{\Theta} P=(\rho+P) u^{3} \Gamma^{3}{ }_{32} u_{3}+(\rho+P) u^{4} \Gamma^{4}{ }_{42} u_{4} .
$$

Substituting (2.12), (2.14), (2.15) into (2.22) we get

$\partial_{\Theta} P=(\rho+P) \frac{\cot \Theta \ell^{-2} \Omega_{\phi}^{2} \sin ^{2} \Theta}{1-\ell^{-2} \Omega_{\phi}^{2} \sin ^{2} \Theta-\ell^{-2} \Omega_{\psi}^{2} \cos ^{2} \Theta}-(\rho+P) \frac{\tan \Theta \ell^{-2} \Omega_{\psi}^{2} \cos ^{2} \Theta}{1-\ell^{-2} \Omega_{\phi}^{2} \sin ^{2} \Theta-\ell^{-2} \Omega_{\psi}^{2} \cos ^{2} \Theta}$,

finally one can write as follows

$$
\partial_{\Theta} P=(\rho+P) \frac{\left(\Omega_{\phi}^{2}-\Omega_{\psi}^{2}\right) \sin (2 \Theta)}{2 \ell^{2}\left(1-\ell^{-2} \Omega_{\phi}^{2} \sin ^{2} \Theta-\ell^{-2} \Omega_{\psi}^{2} \cos ^{2} \Theta\right)} .
$$

So, particularly we have for the contribution only from $\Omega_{\phi}$

$$
\partial_{\Theta} P=(\rho+P) \frac{\Omega_{\phi}^{2} \sin (2 \Theta)}{2 \ell^{2}\left(1-\ell^{-2} \Omega_{\phi}^{2} \sin ^{2} \Theta\right)},
$$


that is in agreement with our result for the drag force in a 5d Kerr-AdS black holes with one rotational parameter and the prediction from [32]. If $\Omega_{\phi}=\Omega_{\psi}$ we get

$$
\partial_{\Theta} P=0
$$

Below we will show that it matches with the holographic calculations for the case with two equal rotational parameters. Now taking into account

$$
\rho+P=s T
$$

the entropy density can be written as

$$
s=\left(\frac{\pi T}{\ell}\right)^{3} \frac{1}{4 G_{5}\left(1-a^{2}\right)}, \quad s \sim\left(\frac{\pi T}{\ell}\right)^{3} \frac{1}{4 G_{5}}
$$

where the Newton's constant equals $G_{5}=\frac{\pi}{\left(2 N_{c}^{2} \ell^{3}\right)}$. A relativistic pressure gradient force is given by

$$
\frac{d p_{\theta}}{d t}=-3 m_{\mathrm{rest}} \frac{\partial_{\theta} P}{s T}
$$

So we can write

$$
\frac{d p_{\theta}}{d t}=-3 m_{\mathrm{rest}} \frac{2}{\pi^{2} T^{4} N_{c}^{2}} \partial_{\theta} P
$$

which is in good agreement with the calculation for the drag force considering a curved string in the 5d Kerr-AdS with one non-zero rotational parameter presented in the next section. We note that $m_{\text {rest }}$ is related with a cut-off at the point $r_{m}$ (where $r_{m}$ is a location of a quark).

\subsection{Drag forces and special Wilson loops}

It has been noticed $[43,63]$ that holographic calculations of the drag forces for an isotropic matter are directly related with calculations of spatial Wilson loops. This relation is also inherited for non-isotropic case including full anisotropic case [50, 62].

Holographic consideration of drag forces in spherically symmetric backgrounds brings with it a new feature that we would like to note before we are going to special calculations. First of all, we note that, by analogy with the case of a flat boundary, it is natural to expect the connection between the technique of suspended strings and the calculation of the spatial Wilson loops. In spherical symmetric backgrounds we deal with boundaries that have $\mathbb{R} \times \mathbb{S}^{3}$ topology. In these cases it is more natural to deal not with rectangular special Wilson loops, but with circular ones. Circular Wilson loops have been considered in holographic approach [64] and refs. therein, however there are obvious obstacles to deal with these objects on the lattice.

In figure 1 we present the geometry of suspected string in $\mathbb{R}^{1} \times \mathbb{S}^{2}$ case where $\mathbb{S}^{2}$ is parametrized by two spherical angles $\phi, \theta$ (in addition, it can be assumed that there is a third angle $\psi$, used for the parameterization of $\mathbb{S}^{3}$, is suppressed in figure 1). 


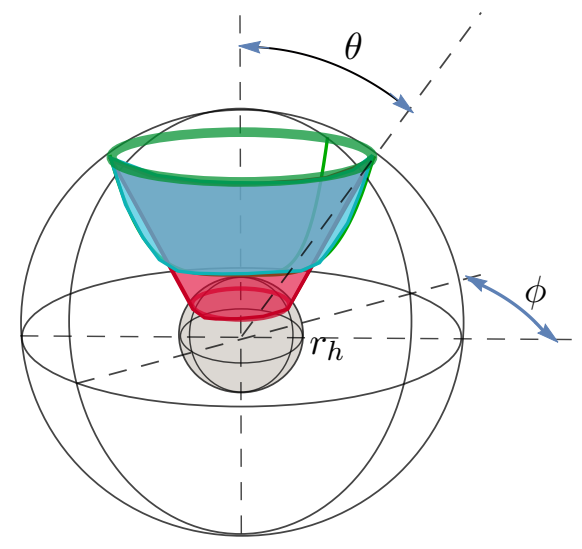

A

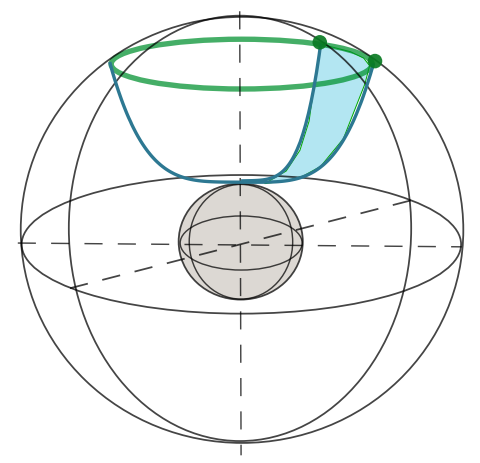

B

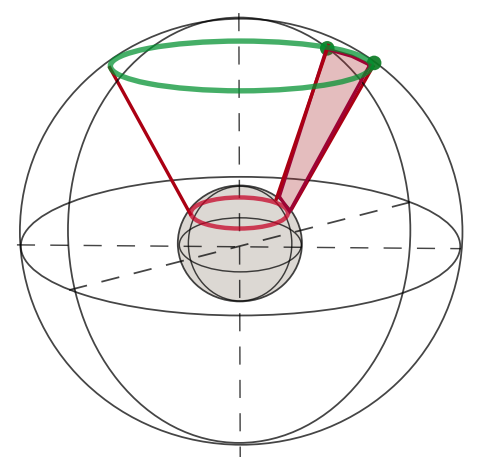

C

Figure 1. Heavy quark moves on the boundary of $A d S_{5}$ having topology $\mathbb{R}^{1} \times \mathbb{S}^{3}\left(\mathbb{R}^{1}\right.$ and the third special coordinate, the angle $\psi$ are suppressed). It moves along a circle with fixed $\theta_{0}$ and varying $\phi$. A) We consider the Wilson loop fixing $\theta=\theta_{0}$ and $0 \leq \phi \leq 2 \pi$ (the small green circle). The blue surface is an extremal surface that ends on trajectory of a heavy quark and touches the horizon of a black hole. The red surface is an analog of the disconnected holographic surface for planar BH calculations. B) The string suspected from the boundary and touched the horizon. C) The straight string ending on the horizon.

\section{$2.4 D=5$ Kerr-AdS black holes}

Our starting point is the following five-dimensional gravity model with a negative cosmological term:

$$
S=-\frac{1}{16 \pi G_{5}} \int \mathrm{d}^{5} x \sqrt{|G|}\left(R_{g}+12 \ell^{2}\right)
$$

where $G_{5}$ is the five-dimensional Newton constant, $G=\operatorname{det} G_{\mu \nu}, G_{\mu \nu}$ being the spacetime metric, and the cosmological constant is $\Lambda=-6 \ell^{2}$. The Einstein equations following from (2.31) are given by

$$
R_{\mu \nu}=-4 \ell^{2} G_{\mu \nu}
$$


Rotating black holes with an AdS aymptotics solve eqs. (2.32). It is known that a rotating black hole in five dimensions is characterized by the mass and two angular parameters related to Casimir invariants of $\mathrm{SO}(4)$. The generic five-dimensional Kerr-AdS metric with two non-zero rotational parameters in the Boyer-Lindquist coordinates is [24]

$$
\begin{aligned}
d s^{2}= & -\frac{\Delta_{r}}{\rho^{2}}\left(d t-\frac{a \sin ^{2} \theta}{\Xi_{a}} d \phi-\frac{b \cos ^{2} \theta}{\Xi_{b}} d \psi\right)^{2}+\frac{\Delta_{\theta} \sin ^{2} \theta}{\rho^{2}}\left(a d t-\frac{\left(r^{2}+a^{2}\right)}{\Xi_{a}} d \phi\right)^{2} \\
& +\frac{\Delta_{\theta} \cos ^{2} \theta}{\rho^{2}}\left(b d t-\frac{\left(r^{2}+b^{2}\right)}{\Xi_{b}} d \psi\right)^{2}+\frac{\rho^{2}}{\Delta_{r}} d r^{2}+\frac{\rho^{2}}{\Delta_{\theta}} d \theta^{2} \\
& +\frac{\left(1+r^{2} \ell^{2}\right)}{r^{2} \rho^{2}}\left(a b d t-\frac{b\left(r^{2}+a^{2}\right) \sin ^{2} \theta}{\Xi_{a}} d \phi-\frac{a\left(r^{2}+b^{2}\right) \cos ^{2} \theta}{\Xi_{b}} d \psi\right)^{2}
\end{aligned}
$$

where $0 \leq \phi, \psi \leq 2 \pi, 0 \leq \theta \leq \pi / 2$, and the parameter $M$ is associated with the mass, $a, b$ are related to the angular momentum and we also have

$$
\begin{aligned}
\Delta_{r} & =\frac{1}{r^{2}}\left(r^{2}+a^{2}\right)\left(r^{2}+b^{2}\right)\left(1+r^{2} \ell^{2}\right)-2 M, \\
\Delta_{\theta} & =\left(1-a^{2} \ell^{2} \cos ^{2} \theta-b^{2} \ell^{2} \sin ^{2} \theta\right), \\
\rho^{2} & =\left(r^{2}+a^{2} \cos ^{2} \theta+b^{2} \sin ^{2} \theta\right), \\
\Xi_{a} & =\left(1-a^{2} \ell^{2}\right), \quad \Xi_{b}=\left(1-b^{2} \ell^{2}\right) .
\end{aligned}
$$

We note that we use Hopf coordinates for the spherical part of the metric (2.33). The horizon position is defined as a largest root $r_{+}$to the equation $\Delta_{r}=0$. The rotational parameters $a$ and $b$ are constrained such that $a^{2}, b^{2} \leq \ell^{-2}$ and the angular momenta [29] are given by

$$
J_{a}=\frac{\pi M a}{2 \Xi_{a}^{2} \Xi_{b}}, \quad J_{b}=\frac{\pi M b}{2 \Xi_{b}^{2} \Xi_{a}} .
$$

The Hawking temperature is defined as

$$
T_{H}=\frac{1}{2 \pi}\left(r_{+}\left(1+r_{+}^{2} \ell^{2}\right)\left(\frac{1}{r_{+}^{2}+a^{2}}+\frac{1}{r_{+}^{2}+b^{2}}\right)-\frac{1}{r_{+}}\right) .
$$

The dependence of $r_{+}$on parameters $a$ and $b$ is illustrated in figures 2 and 3 .

We note that in the Boyer-Lindquist coordinates, the metric is asymptotic to $A d S_{5}$ in a rotating frame, with angular velocities

$$
\Omega_{\phi}^{\infty}=-a \ell^{2}, \quad \Omega_{\psi}^{\infty}=-b \ell^{2}
$$

Let us consider the case of the Kerr-AdS solutions with $a=b$, then the metric (2.33) takes 


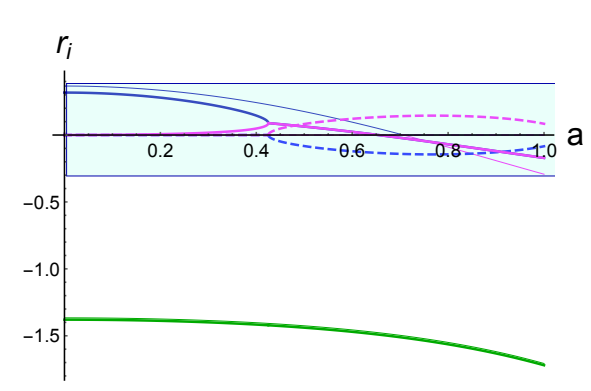

A

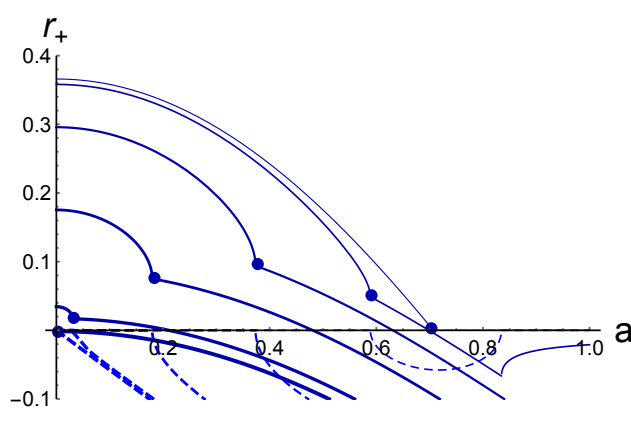

$\mathrm{C}$
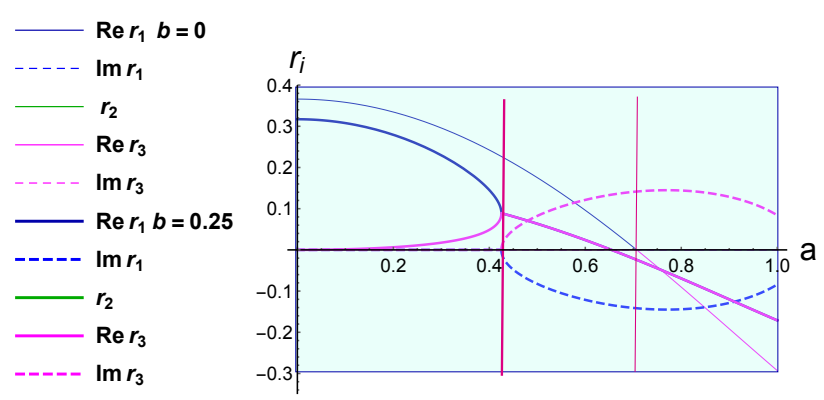

$\mathrm{B}$

Figure 2. A) 3 roots of equation $\Delta(r)=0$ vs $a$ for $b=0$ (thin lines) and $b=0.2$ (thick lines). Dashed line shows the imaginary part of the first root $r_{+}$. B) First two roots for the same values of $b=0,0.2$. C) The first root vs $a$ for variety of $b$. We see that only for $a<a_{c r}(b)$ the root $r_{1}$ is positive, $a_{c r}(0)=1 / \sqrt{2}$. D) The dependence of critical points of $a_{c r}$ on $b$. Here $M$ is taken to be 0.25 and $\ell=1$.

the form

$$
\begin{aligned}
d s^{2}= & -\left(1+\rho^{2} \ell^{2}-\frac{2 M}{\rho^{2}}\right) d t^{2}+\frac{\rho^{2}}{\Delta_{r}} d r^{2}+\frac{\rho^{2}}{\Delta_{\theta}} d \theta^{2} \\
& +\frac{\sin ^{2} \theta}{\Xi^{2}}\left(\rho^{2} \Xi+\frac{2 a^{2} M}{\rho^{2}} \sin ^{2} \theta\right) d \phi^{2}+\frac{\cos ^{2} \theta}{\Xi^{2}}\left(\Xi \rho^{2}+\frac{2 a^{2} M \cos ^{2} \theta}{\rho^{2}}\right) d \psi^{2} \\
& +\frac{2 a \sin ^{2} \theta}{\Xi}\left(\rho^{2} \ell^{2}-\frac{2 M}{\rho^{2}}\right) d t d \phi+\frac{2 a \cos ^{2} \theta}{\Xi}\left(\rho^{2} \ell^{2}-\frac{2 M}{\rho^{2}}\right) d t d \psi, \\
& +\frac{4 M a^{2} \sin ^{2} \theta \cos ^{2} \theta}{\Xi^{2} \rho^{2}} d \phi d \psi,
\end{aligned}
$$

where for $(2.35)$ we have

$$
\begin{aligned}
& \Delta_{r}=\frac{1}{r^{2}}\left(r^{2}+a^{2}\right)^{2}\left(1+r^{2} \ell^{2}\right)-2 M, \\
& \Delta_{\theta}=\left(1-a^{2} \ell^{2}\right), \quad \rho^{2}=\left(r^{2}+a^{2}\right), \quad \Xi=\left(1-a^{2} \ell^{2}\right) .
\end{aligned}
$$

The $5 \mathrm{~d}$ Kerr-AdS solution with a single rotational parameter $(a \neq 0, b=0)$ reads

$d s^{2}=-\frac{\Delta_{r}}{\rho^{2}}\left(\mathrm{~d} t-\frac{a}{\Xi_{a}} \sin ^{2} \theta \mathrm{d} \phi\right)^{2}+\frac{\rho^{2}}{\Delta_{r}} \mathrm{~d} r^{2}+\frac{\rho^{2}}{\Delta_{\theta}} \mathrm{d} \theta^{2}+\frac{\Delta_{\theta} \sin ^{2} \theta}{\rho^{2}}\left[a \mathrm{~d} t-\frac{\left(r^{2}+a^{2}\right)}{\Xi_{a}} \mathrm{~d} \phi\right]^{2}+r^{2} \cos ^{2} \theta \mathrm{d} \psi^{2}$, 


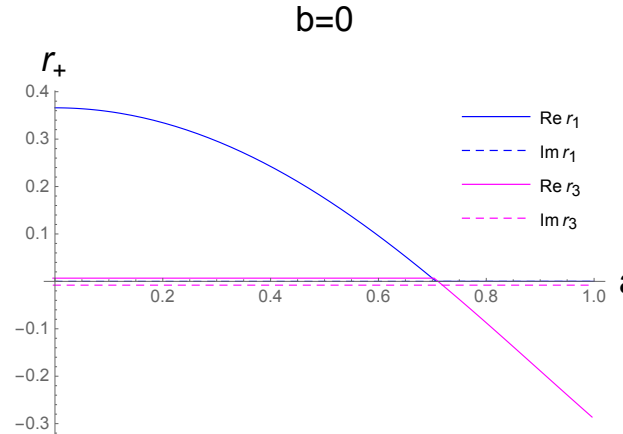

A

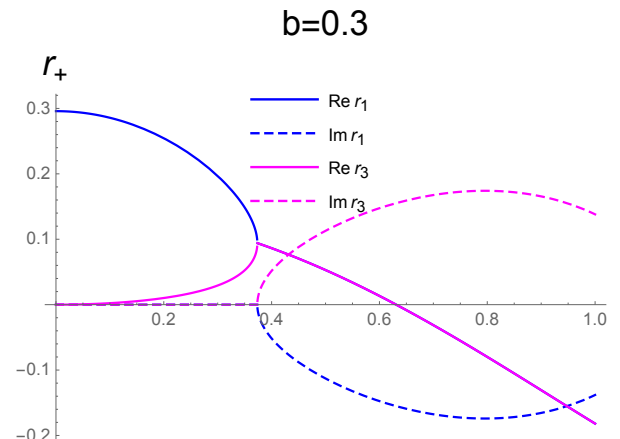

C

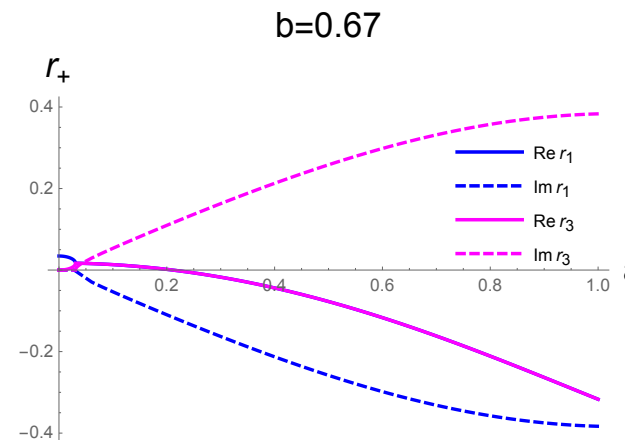

$\mathrm{E}$

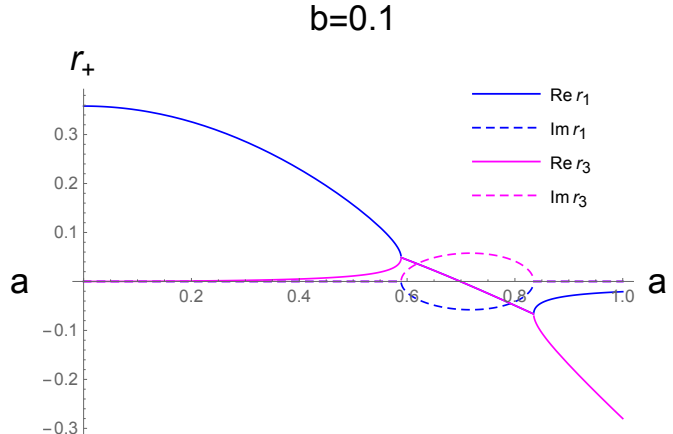

B

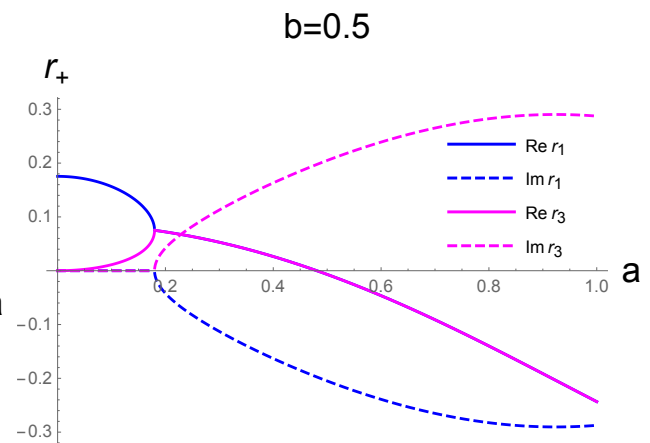

$\mathrm{D}$

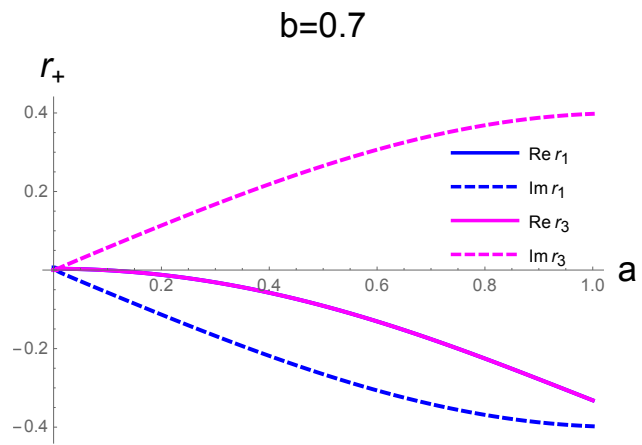

F

Figure 3. 3 roots of equation $\Delta(r)=0$ vs $a$ for varieties of $b$. We see that only the first root can be positive for $a<a(b)$. Here $M$ is taken to be 0.25 and $\ell=1$.

with

$$
\begin{aligned}
& \Delta_{r}=\left(r^{2}+a^{2}\right)\left(1+\ell^{2} r^{2}\right)-2 M, \\
& \Delta_{\theta}=1-a^{2} \ell^{2} \cos ^{2} \theta, \\
& \Xi_{a}=1-a^{2} \ell^{2}, \quad \rho^{2}=r^{2}+a^{2} \cos ^{2} \theta .
\end{aligned}
$$


For the single parameter 5d Kerr-AdS hole the horizon position is written down explicitly

$$
r_{+}=\frac{\sqrt{\sqrt{\left(1-a^{2} \ell^{2}\right)^{2}+8 M \ell^{2}}-\left(1-a^{2} \ell^{2}\right)}}{\sqrt{2} \ell} .
$$

The case of single rotational parameter can this solutions can be constructed from $4 \mathrm{~d}$ case as the stationary asymptotically flat higher dimensional black holes from the work by Myers and Perry [65].

The transformations that convert (2.33) from Boyer-Lindquist coordinates to asymptotically AdS coordinates $a \neq b \neq 0[24]$ are

$$
\begin{aligned}
\Xi_{a} y^{2} \sin ^{2} \Theta & =\left(r^{2}+a^{2}\right) \sin ^{2} \theta, \\
\Xi_{b} y^{2} \cos ^{2} \Theta & =\left(r^{2}+b^{2}\right) \cos ^{2} \theta, \\
\Phi & =\phi+a \ell^{2} t, \\
\Psi & =\psi+b \ell^{2} t, \\
T & =t .
\end{aligned}
$$

It should be noted the coordinates (2.44) are difficult for direct representation of the $5 \mathrm{~d}$ Kerr-AdS metrics, except the case when we have the two non-zero rotational parameters which are equal by its magnitude:

$$
\begin{aligned}
d s^{2}= & -\left(1+y^{2} \ell^{2}\right) d T^{2}+y^{2}\left(d \Theta^{2}+\sin ^{2} \Theta d \Phi^{2}+\cos ^{2} \Theta d \Psi^{2}\right) \\
& +\frac{2 M}{y^{2} \Xi^{3}}\left(d T-a \sin ^{2} \Theta d \Phi-a \cos ^{2} \Theta d \Psi\right)^{2}+\frac{y^{4} d y^{2}}{y^{4}\left(1+y^{2} \ell^{2}\right)-\frac{2 M}{\Xi^{2}} y^{2}+\frac{2 M a^{2}}{\Xi^{3}}} .
\end{aligned}
$$

The position of the horizon of the extremal black hole in these coordinates [24] reads

$$
y^{2}=\frac{1}{4 \Xi}\left[4 a^{2} \ell^{2}-1+\sqrt{1+8 a^{2} \ell^{2}}\right] .
$$

\subsection{Thermodynamics of the 5d Kerr-AdS black holes}

Thermodynamics of the holographic background defines the first order phase transition on ( $T$-parameters) phase diagram. In the gravity language, the origin of this phase transition is related with is a non-trivial dependence of the Hawking temperature on the location of the horizon. A typical example of such dependence is given by the Van-Der-Waals curve. Here we are going to consider the angular momentum of the rotating medium as physical parameters and find out how they affect on the phase diagram. Technically this means that we have to understand a location of the phase transition of the background in a two dimensional space $(T-a)$ for the case of one rotational parameter $a$ ( $T$ is the Hawking temperature given by (2.36)) and in three dimensional space $(T-a-b)$ for the case of two rotational parameters, $a$ and $b$.

Below we illustrate that the Kerr-AdS black hole inherits the main thermodynamical property of the AdS black hole - the presence of the Hawking-Page phase transition. For this purpose we trace the dependence of the Hawking temperature (2.36) on the rotational parameters $a$ and $b$ at fixed value of the horizon $r_{+}$. We present this in figure 4, using ContourPlot. The factor 2.9 ensures that the background phase transition for $a=b=0$ is at $T_{c r}(0,0)=0.160 \mathrm{GeV}$, that is in agreement with lattice calculations and LHC and 

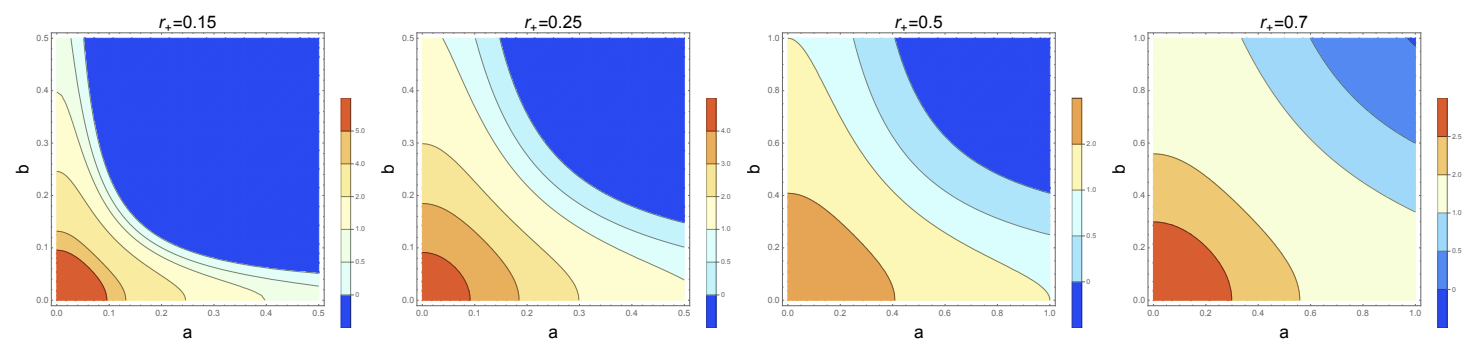

Figure 4. Contour plot of the Hawking temperature (2.36) multiplied by $2 \pi \cdot 2.9$ depending on $a, b$ and $r_{+}$.

RHIC data. From (2.36) it follows that the dependence of the horizon on the temperature is multivalued. To illustrate this we draw $T$ vs $r_{+}$for different values of $a$ and $b$, see the left column of figure 5. From these plots we observe that depending on the values of $a$ and $b$ the background allows to have the Van-Der-Waals type of the temperature dependence on $r_{+}$and for some cases the temperature becomes a three-valued function on the horizon $r_{+}$. To show that the Hawking-Page transition takes place for the Kerr-AdS black hole, we have to consider the dependence of free energy $F$ on temperature $T$. To this purpose we find the dependence of the entropy $S$ on $T$

$$
S=\frac{\pi^{2}}{2 \Xi_{a} \cdot \Xi_{b}} \frac{\left(r_{+}^{2}+a^{2}\right)\left(r_{+}^{2}+b^{2}\right)}{r_{+}}
$$

where $\Xi_{a}=1-a^{2} \ell^{2}$ and $\Xi_{b}=1-a^{2} \ell^{2}$.

From the middle column of figure 5 we see that for small enough values of $a$ and/or $b$, there is a region of temperatures at which the entropy is three-valued. If we will increase the value of one of the rotational parameters this region disappears and for the certain temperature the entropy becomes only one valued.

In the right column of figure 5 we show the influence of the rotational parameters on the dependence of the free energy on the temperature. For the free energy we use the following relation

$$
F(r)=\int_{r}^{r_{+}} S(x) T^{\prime}(x) d x
$$

Again for the small values of $a$ and $b$ we observe that the free energy is multivalued and there are swallow tails in $(F-T)$ plane and the first order phase transition takes place. At some points, $\left(a_{c r}, b_{c r}\right)$, discontinuity of $F$ disappears and the first order phase transition ends.

Finally, in figure $6 \mathbf{A}$ we show how the rotational parameters affect on the value of the temperature of the phase transition. The higher the value of the rotational parameters we have, the lower temperature at which the phase transition we get. So we see that it's quite justified to consider a case with one non-zero rotational parameter. In figure $6 \mathbf{B}$ we illustrate the location of the transition plane $T_{c r}=T_{c r}(a, b)$. 

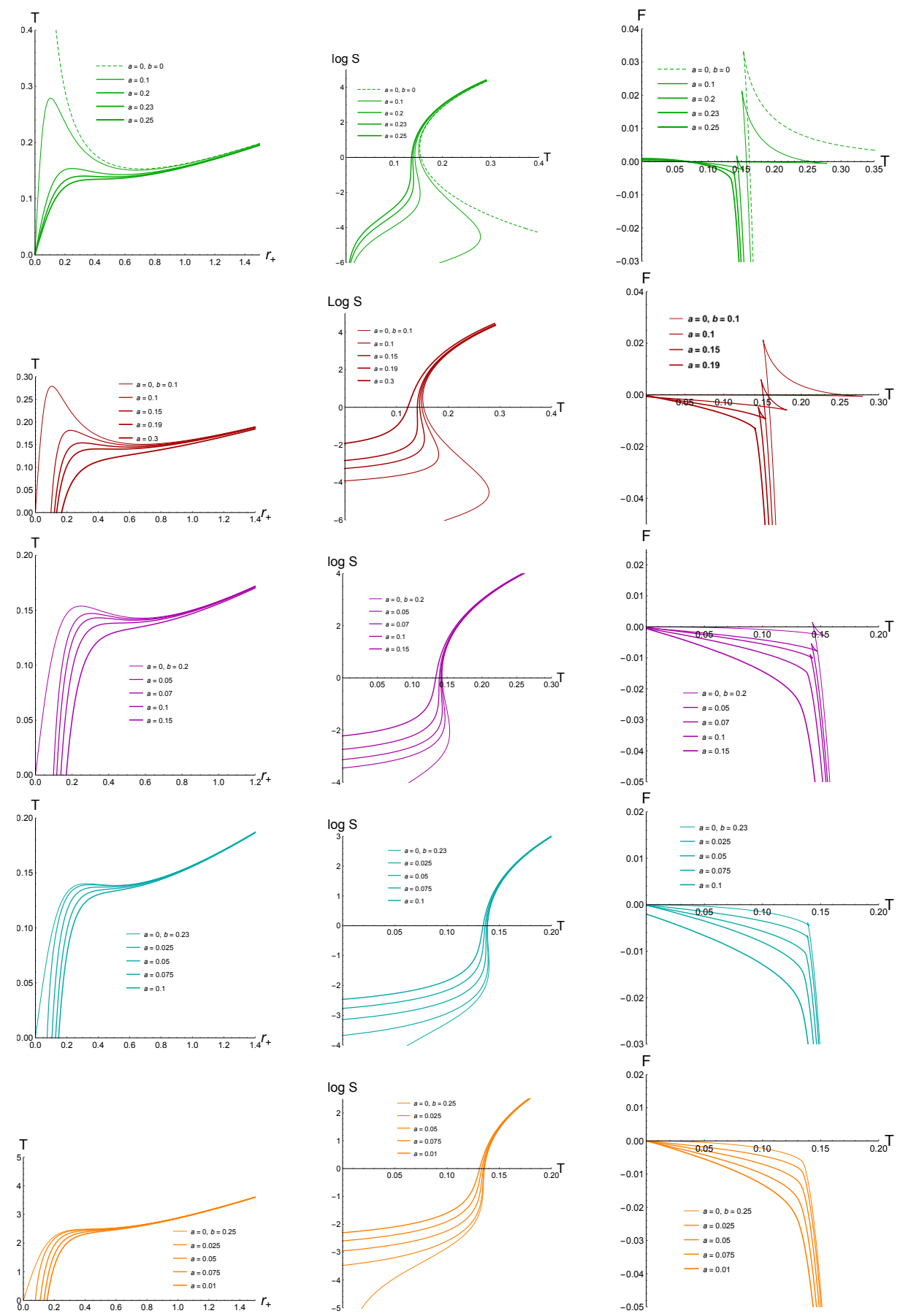

Figure 5. The plots in the first column show the dependence of temperature on $r_{h}$ for varieties $a$ and $b$. The plots in the second column show a logarithmic dependence of entropy on temperature. The plots in the third column show the dependence of free energy on temperature. 


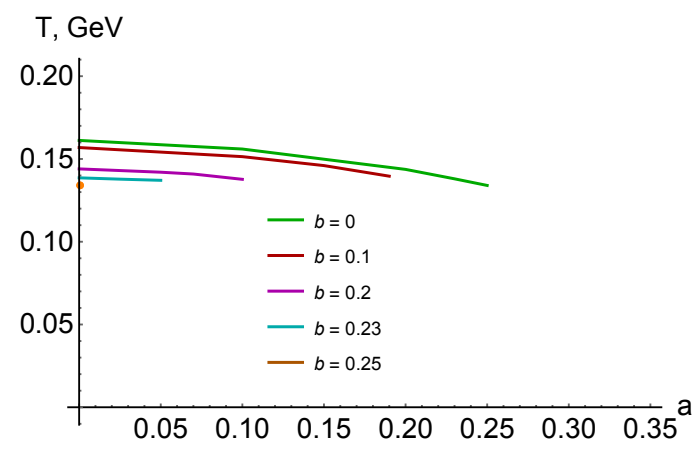

A

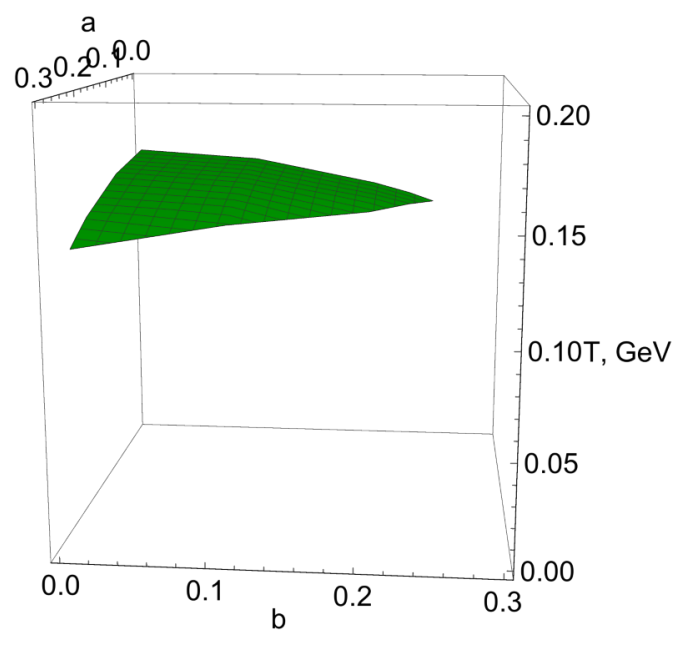

$\mathrm{B}$

Figure 6. Locations of the first order phase transition in terms of $a$ and $b$. A) Locations of the end points for fixed $b$ are indicated by dots. We see that all end points have the same temperature $T_{C E P}=0.134 \mathrm{GeV}$. B) The location of the phase transition $T_{c r}=T_{c r}\left(a^{2}, b^{2}\right)$ is shown by the green surface.

\section{Drag force in 5d Kerr-AdS background with one rotational parameter}

\subsection{Static curved string in the 5d Kerr-AdS background}

Now we turn to discussion of a curved string in the 5d Kerr-AdS background with one nonzero rotational parameter $(a \neq 0, b=0)$. We use the form of the metric in Boyer-Lindquist coordinates $(2.41)-(2.42) .^{2}$ The string worldsheet is parametrized as $\left(\sigma^{0}, \sigma^{1}\right)=(t, r)$. The embedding is characterized by

$$
\theta=\theta(t, r), \quad \phi=\phi(t, r), \quad \psi=\psi(t, r) .
$$

Then non-zero components of the induced metric $g_{\alpha \beta}(2.2)$ look like

$$
\begin{aligned}
g_{t t} & =G_{t t}+2 G_{t \phi} \dot{\phi}+G_{\phi \phi} \dot{\phi}^{2}+G_{\theta \theta} \dot{\theta}^{2}+G_{\psi \psi} \dot{\psi}^{2}, \\
g_{r r} & =G_{r r}+G_{\phi \phi} \phi^{\prime 2}+G_{\theta \theta} \theta^{\prime 2}+G_{\psi \psi} \psi^{\prime 2}, \\
g_{r t} & =G_{t \phi} \phi^{\prime}+G_{\theta \theta} \theta^{\prime} \dot{\theta}+G_{\phi \phi} \phi^{\prime} \dot{\phi}+G_{\psi \psi} \dot{\psi} \psi^{\prime},
\end{aligned}
$$

where $G_{\mu \nu}$ are components of the 5 d Kerr-AdS metric (2.41)-(2.42) and we define ${ }^{\cdot}=\frac{d}{d t} A$, $'=\frac{d}{d r} A$.

The equations of motion that follow from the Nambu-Goto action calculated with (3.2)(3.4) seems to be quite difficult to work, instead of them we will consider the linearized string equations of motion for small values of the rotational parameter $a$. For the ansatz

\footnotetext{
${ }^{2}$ See the SageMath notebook https://cocalc.com/share/565d762758a554b6adffa16d5562e097e0e565eb/ Kerr-AdS-5D-string-b_zero.ipynb for details on the calculation.
} 
of the curved string solution we take an expansion by order in $a$ as follows

$$
\begin{aligned}
\phi(t, r) & =\Phi_{0}+\beta_{1} a \ell^{2} t+\beta_{1} a \phi_{1}(r)+\mathcal{O}\left(a^{2}\right), \\
\theta(r) & =\Theta_{0}+a^{2} \theta_{1}(r)+\mathcal{O}\left(a^{4}\right), \\
\psi(t, r) & =\Psi_{0}+\beta_{2} a \ell^{2} t+\beta_{2} a \psi_{1}(r)+\mathcal{O}\left(a^{2}\right),
\end{aligned}
$$

where $\beta_{1}$ and $\beta_{2}$ are some constants such that $\beta_{1}^{2}+\beta_{2}^{2}=1$. We note that with $\beta_{2}=0$ we come to the case with fixed $\psi$, i.e. $\psi(t, r)=\Psi_{0}$.

Correspondingly, the induced metric (B.15) with plugged (3.5) into (3.2)-(3.4) takes the form

$$
\begin{aligned}
-g= & \left(\left(a \Delta_{r}-a\left(r^{2}+a^{2}\right) \Delta_{\theta}\right) \frac{\sin ^{2} \theta}{\Xi_{a} \rho^{2}} a \beta_{1} \phi_{1}^{\prime}+\right. \\
& \left.+\left(\Delta_{\theta}\left(r^{2}+a^{2}\right)^{2}-a^{2} \Delta_{r} \sin ^{2} \theta\right) \frac{\sin ^{2} \theta}{\Xi_{a}^{2} \rho^{2}} a^{2} \beta_{1}^{2} \ell^{2} \phi_{1}^{\prime}+a^{2} \beta_{2}^{2} \ell^{2} r^{2} \cos ^{2} \theta \psi_{1}^{\prime}\right)^{2} \\
- & \left(\frac{\rho^{2}}{\Delta_{r}}+\frac{\rho^{2}}{\Delta_{\theta}} a^{4} \theta_{1}^{\prime 2}+\left(\Delta_{\theta}\left(r^{2}+a^{2}\right)^{2}-a^{2} \Delta_{r} \sin ^{2} \theta\right) \frac{\sin ^{2} \theta}{\Xi_{a}^{2} \rho^{2}} a^{2} \beta_{1}^{2} \phi_{1}^{\prime 2}+a^{2} \beta_{2}^{2} r^{2} \cos ^{2} \theta \psi_{1}^{\prime 2}\right) \\
\times & \left(\left(a^{2} \Delta_{\theta} \sin ^{2} \theta-\Delta_{r}\right) \frac{1}{\rho^{2}}+\left(a \Delta_{r}-a\left(r^{2}+a^{2}\right) \Delta_{\theta}\right) \frac{2 \sin ^{2} \theta}{\Xi_{a} \rho^{2}} a \beta_{1} \ell^{2}\right. \\
& \left.\quad+\left(\Delta_{\theta}\left(r^{2}+a^{2}\right)^{2}-a^{2} \Delta_{r} \sin ^{2} \theta\right) \frac{\sin ^{2} \theta}{\Xi_{a}^{2} \rho^{2}} a^{2} \beta_{1}^{2} \ell^{4}+r^{2} \cos ^{2} \theta a^{2} \beta_{2}^{2} \ell^{4}\right)
\end{aligned}
$$

From the Nambu-Goto action (2.1) with (3.6) we can find out the first integrals expanded in series by $a$ and the following relations hold

$$
\phi_{1}(r)=\mathfrak{p} \int_{r_{+}}^{r} \frac{d \bar{r}}{\bar{r}^{4} h(\bar{r})}, \quad \psi_{1}(r)=\mathfrak{q} \int_{r_{+}}^{r} \frac{d \bar{r}}{\bar{r}^{4} h(\bar{r})},
$$

where $\mathfrak{p}$ and $\mathfrak{q}$ are some constants and $h(r)$ is

$$
h(r)=\ell^{2}+\frac{1}{r^{2}}-\frac{2 M}{r^{4}},
$$

which is actually the blackening factor for the $5 \mathrm{~d}$ AdS-Schwarzschild black hole. It worth to be noted that the appearance (3.8) is not surprising since we work with an expansion with the small rotational parameter $a$ and the Kerr-AdS black hole asymptotes to the AdS-Schwarzschild black hole (A.1).

Substituting (3.7) into the Nambu-Goto action (2.1) with (3.6) we derive the linearized equation of motion for $\theta_{1}$ in the following form

$$
\Upsilon^{\prime}+\frac{2\left(r+2 \ell^{2} r^{3}\right)}{r^{4} h} \Upsilon+\frac{1+\beta_{2}^{2} \mathfrak{q}^{2}-4 \ell^{2} \beta_{1} M-\beta_{1}^{2} \mathfrak{p}^{2}+\left(2 \beta_{1}+\beta_{1}^{2}-\beta_{2}^{2}\right) \ell^{4} r^{4}}{2 r^{8} h^{2}} \sin \left(2 \Theta_{0}\right)=0,
$$

where we reduce the second order differential equation to a first one by the change of the variable $\Upsilon=\theta_{1}^{\prime}$.

Taking into account the dimensions of the quantities $[M] \sim r^{2},[\ell] \sim \frac{1}{r},[h] \sim \frac{1}{r^{2}}$, $\left[\theta_{1}\right] \sim \frac{1}{r^{2}}$ one sees that all terms in the l.h.s. of the expression (3.9) have dimension $\frac{1}{r^{4}}$. 
The solution to eq. (3.9) can be represented in the following form

$$
\begin{aligned}
& \theta_{1}^{\prime}=\frac{C_{1}}{r^{4} h}-\frac{\sin \left(2 \Theta_{0}\right)}{2 r^{4} h}\left(\beta_{1}^{2}-\beta_{2}^{2}+2 \beta_{1}\right) \ell^{2} r-\frac{\ell \sin \left(2 \Theta_{0}\right)}{2 \sqrt{2} \sqrt{1+8 M \ell^{2}} r^{4} h} \tan ^{-1}\left(\frac{\sqrt{2} \ell r}{\sqrt{1-\sqrt{1+8 M \ell^{2}}}}\right) \\
& \times \frac{\left(4 \ell^{2} M+1-\sqrt{1+8 M \ell^{2}}\right)\left(\beta_{1}^{2}-\beta_{2}^{2}\right)-2\left(\beta_{1}^{2} \mathfrak{p}^{2}-1-\beta_{2}^{2} \mathfrak{q}^{2}\right)-2 \beta_{1}\left(\sqrt{1+8 M \ell^{2}}-1\right)}{\sqrt{1-\sqrt{1+8 M \ell^{2}}}} \\
& +\frac{\ell \sin \left(2 \Theta_{0}\right)}{2 \sqrt{2} \sqrt{1+8 M \ell^{2}} r^{4} h} \tan ^{-1}\left(\frac{\sqrt{2} \ell r}{\sqrt{1+\sqrt{1+8 M \ell^{2}}}}\right) \\
& \times \frac{\left(4 \ell^{2} M+1+\sqrt{1+8 \ell^{2} M}\right)\left(\beta_{1}^{2}-\beta_{2}^{2}\right)-2\left(\beta_{1}^{2} \mathfrak{p}^{2}-1-\beta_{2}^{2} \mathfrak{q}^{2}\right)+2 \beta_{1}\left(1+\sqrt{1+8 M \ell^{2}}\right)}{\sqrt{1+\sqrt{1+8 M \ell^{2}}}}
\end{aligned}
$$

where $C_{1}$ is a constant of integration. Remembering that in eq. (3.9) it appears a contribution of the AdS-Schwarzschild blackening function (3.8) one can use the relations for the horizon and the Hawking temperature by (A.2)-(A.4) and represent the 1.h.s. of (3.10) in terms of $r_{H}$

$$
\begin{aligned}
& \theta_{1}^{\prime}=\frac{\tilde{C}_{1}}{r^{4} h}-\frac{\sin \left(2 \Theta_{0}\right)}{2 r^{4} h}\left(\beta_{1}^{2}-\beta_{2}^{2}+2 \beta_{1}\right) \ell^{2} r \\
& +\frac{\sin \left(2 \Theta_{0}\right)}{2\left(2 \ell^{2} r_{H}^{2}+1\right) r^{4} h} \log \left(\frac{r+r_{H}}{r-r_{H}}\right) \frac{\ell^{4} r_{H}^{4}\left(\beta_{1}^{2}-\beta_{2}^{2}\right)-\left(\beta_{1}^{2} \mathfrak{p}^{2}-1-\beta_{2}^{2} \mathfrak{q}^{2}\right)-2 \beta_{1} \ell^{2} r_{H}^{2}}{r_{H}} \\
& +\frac{\ell \sin \left(2 \Theta_{0}\right)}{2\left(2 \ell^{2} r_{H}^{2}+1\right) r^{4} h} \tan ^{-1}\left(\frac{\ell r}{\sqrt{\ell^{2} r_{H}^{2}+1}}\right) \frac{\left(\ell^{2} r_{H}^{2}+1\right)^{2}\left(\beta_{1}^{2}-\beta_{2}^{2}\right)-\left(\beta_{1}^{2} \mathfrak{p}^{2}-1-\beta_{2}^{2} \mathfrak{q}^{2}\right)+2 \beta_{1}\left(r_{H}^{2} \ell^{2}+1\right)}{\sqrt{r_{H}^{2} \ell^{2}+1}}
\end{aligned}
$$

where we also take into account that $\frac{1}{i} \tan ^{-1}\left(\frac{x}{i}\right)=-\tanh ^{-1} x$ and $\tanh ^{-1} x=\frac{1}{2} \log \left(\frac{1+x}{1-x}\right)$. Since the solutions to angular variables are known (3.7), (3.11), we are able to write down the conjugate momenta with respect to (2.4) are

$$
\begin{aligned}
\pi_{\theta}^{r} & =h(r) r^{4} \theta_{1}^{\prime} a^{2}+\mathcal{O}\left(a^{4}\right), \\
\pi_{\phi}^{r} & =h(r) r^{4} \phi_{1}^{\prime} \sin ^{2}\left(\Theta_{0}\right) a+\mathcal{O}\left(a^{2}\right), \\
\pi_{\psi}^{r} & =h(r) r^{4} \psi_{1}^{\prime} \cos ^{2}\left(\Theta_{0}\right) a+\mathcal{O}\left(a^{2}\right) .
\end{aligned}
$$

Expanding (3.12) with (3.7), (3.11) near the boundary $r \rightarrow+\infty$ we get the following relations for the conjugate momenta

$$
\begin{aligned}
\pi_{\theta}^{r}= & \left(\frac{2 \tilde{C}_{1}}{\sin \left(2 \Theta_{0}\right)}-\left(\beta_{1}^{2}-\beta_{2}^{2}+2 \beta_{1}\right) \ell^{2} r-\frac{\left(\beta_{1}^{2}-\beta_{2}^{2}+2 \beta_{1}\right)}{r}\right. \\
& \left.+\frac{\pi \ell}{2\left(2 \ell^{2} r_{H}^{2}+1\right)} \frac{\left(\ell^{2} r_{H}^{2}+1\right)^{2}\left(\beta_{1}^{2}-\beta_{2}^{2}\right)+2 \beta_{1}\left(r_{H}^{2} \ell^{2}+1\right)-\left(\beta_{1}^{2} \mathfrak{p}^{2}-1-\beta_{2}^{2} \mathfrak{q}^{2}\right)}{\sqrt{r_{H}^{2} \ell^{2}+1}}\right) \sin \left(2 \Theta_{0}\right) \frac{a^{2}}{2} \\
& +\mathcal{O}\left(a^{2}\right) \\
\pi_{\phi}^{r}= & \mathfrak{p} \sin \left(\Theta_{0}\right)^{2} a+\mathcal{O}\left(a^{2}\right) \\
\pi_{\psi}^{r}= & \mathfrak{q} \cos \left(\Theta_{0}\right)^{2} a+\mathcal{O}\left(a^{2}\right) .
\end{aligned}
$$

where we use $\log \left(\frac{r+r_{H}}{r-r_{H}}\right) \approx 2 \frac{r_{H}}{r}$ and $\tan ^{-1}\left(\frac{r \ell}{\sqrt{\ell^{2} r_{H}^{2}+1}}\right) \approx \frac{\pi}{2}-\frac{\sqrt{\ell^{2} r_{H}^{2}+1}}{\ell r}$. 
The components of the drag force can be found owing to (2.9) and (3.13)-(3.15) as

$$
\begin{aligned}
\frac{d p_{\theta}}{d t}= & \left(-\frac{2 \tilde{C}_{1}}{\sin \left(2 \Theta_{0}\right)}+\left(\beta_{1}^{2}-\beta_{2}^{2}+2 \beta_{1}\right) \ell^{2} r+\frac{\left(\beta_{1}^{2}-\beta_{2}^{2}+2 \beta_{1}\right)}{r}\right. \\
& \left.-\frac{\pi \ell}{2\left(2 \ell^{2} r_{H}^{2}+1\right)} \frac{\left(\ell^{2} r_{H}^{2}+1\right)^{2}\left(\beta_{1}^{2}-\beta_{2}^{2}\right)+2 \beta_{1}\left(r_{H}^{2} \ell^{2}+1\right)-\left(\beta_{1}^{2} \mathfrak{p}^{2}-1-\beta_{2}^{2} \mathfrak{q}^{2}\right)}{\sqrt{r_{H}^{2} \ell^{2}+1}}\right) \frac{\sin \left(2 \Theta_{0}\right)}{4 \pi \alpha^{\prime}} a^{2} \\
\frac{d p_{\phi}}{d t}= & -\frac{1}{2 \pi \alpha^{\prime}} \mathfrak{p} \sin \left(\Theta_{0}\right)^{2} a+\mathcal{O}\left(a^{2}\right) \\
\frac{d p_{\psi}}{d t}= & -\frac{1}{2 \pi \alpha^{\prime}} \mathfrak{q} \cos \left(\Theta_{0}\right)^{2} a+\mathcal{O}\left(a^{2}\right) .
\end{aligned}
$$

We see that the component $\frac{d p_{\theta}}{d t}(3.16)$ has a linearly divergent term with $r \rightarrow \infty$

$$
\frac{d p_{\theta}}{d t}=\mathfrak{B}_{1}(\mathfrak{b}) \ell^{2} r \frac{\sin \left(2 \Theta_{0}\right)}{2 \pi \alpha^{\prime}} a^{2}+\ldots,
$$

where $\mathfrak{B}_{1}(\mathfrak{b})=\beta_{1}^{2}-\beta_{2}^{2}+2 \beta_{1}$ and we use parametrization $\beta_{1}=\sin \mathfrak{b}, \beta_{2}=\cos \mathfrak{b}$, so $\mathfrak{B}_{1}(\mathfrak{b})=2 \sin (\mathfrak{b})-\cos (2 \mathfrak{b})$. The term in r.h.s. of (3.19) can be associated to an infinite mass of the heavy quark [39]. One can renormalize it introducing a cut-off $r_{m}$. By virtue of (A.4) we get from (3.19)

$$
\frac{d p_{\theta}}{d t}=\mathfrak{B}_{1}(\mathfrak{b})\left(\ell^{2} m_{\text {rest }}+\frac{1}{4 \pi \alpha^{\prime}}\left(\pi T_{H}+\sqrt{\pi^{2} T_{H}^{2}-2 \ell^{2}}\right)\right) \frac{a^{2}}{2} \sin \left(2 \Theta_{0}\right),+\ldots
$$

where we take for the cut-off $r_{m}=2 \pi \alpha^{\prime} m_{\text {rest }}$.

It is interesting to note that $\mathfrak{B}_{1}(\mathfrak{b})$ can have different signs. Here the sign "-" shows that the drag force is opposite the quark movement.

With respect to values of parameter $(\mathfrak{b})$ we have the following special cases

- $\mathfrak{b}=\pi / 2, \mathfrak{B}_{1}(\mathfrak{b})=3$.

The dependence of $\frac{d p_{\theta}}{d t}$ on the temperature is

$$
\begin{aligned}
\frac{d p_{\theta}}{d t} & =\left(6 \ell^{2} m_{\text {rest }}+\frac{3}{2 \pi \alpha^{\prime}}\left(\pi T_{H}+\sqrt{\pi^{2} T_{H}^{2}-2 \ell^{2}}\right)\right) \frac{a^{2}}{4} \sin \left(2 \Theta_{0}\right)+\mathcal{O}\left(a^{2}\right) \\
\frac{d p_{\phi}}{d t} & =-\frac{1}{2 \pi \alpha^{\prime}} \mathfrak{p} \sin \left(\Theta_{0}\right)^{2} a+\mathcal{O}\left(a^{2}\right), \quad \frac{d p_{\psi}}{d t}=0 .
\end{aligned}
$$

This case corresponds to fixed $\psi$ and the conjugate momentum in the $\psi$-direction is equals to 0 and covers the result of [32], i.e. the leading term of the drag force in the $\theta$-direction is $3 \ell^{2} m_{\text {rest }}$.

- $\mathfrak{b}=\pi / 4, \mathfrak{B}_{1}(\mathfrak{b})=\sqrt{2}$,

$$
\begin{aligned}
\frac{d p_{\theta}}{d t}= & \left(-\frac{2 \tilde{C}_{1}}{\sin \left(2 \Theta_{0}\right)}+\sqrt{2} \ell^{2} r+\frac{\sqrt{2}}{r}\right. \\
& \left.-\frac{\pi \ell}{2\left(2 \ell^{2} r_{H}^{2}+1\right)} \frac{\sqrt{2}\left(r_{H}^{2} \ell^{2}+1\right)+1-\frac{1}{2}\left(\mathfrak{p}^{2}-\mathfrak{q}^{2}\right)}{\sqrt{r_{H}^{2} \ell^{2}+1}}\right) \frac{a^{2} \sin \left(2 \Theta_{0}\right)}{2}, \\
\frac{d p_{\phi}}{d t}= & -\frac{1}{2 \pi \alpha^{\prime}} \mathfrak{p} \sin \left(\Theta_{0}\right)^{2} a+\mathcal{O}\left(a^{2}\right) \\
\frac{d p_{\psi}}{d t}= & -\frac{1}{2 \pi \alpha^{\prime}} \mathfrak{q} \sin \left(\Theta_{0}\right)^{2} a+\mathcal{O}\left(a^{2}\right)
\end{aligned}
$$


Here the relations seems to have the same form comparing as the previous case except the coefficients.

- $0 \leq \mathfrak{b} \leq \tan ^{-1}\left(\frac{\sqrt{3}-1}{\sqrt{2} \sqrt[4]{3}}\right) \approx 0.375,-1 \leq \mathfrak{B}_{1}(\mathfrak{b}) \leq 0$.

In this case, the drag force acts on the quark in the opposite direction of the joint motion of the quark and rotating medium. Note that this case is not exhibited in [32].

Eq. (3.13)-(3.18) reflect the drag force for the fixed quark in the rotating QGP, so for the rotating parameter $a=0$ it just vanishes. One can complete this relation supposing that the quark also slowly moves with an angular velocity that has components $\left(\omega_{\theta}, \omega_{\phi}, \omega_{\psi}\right)$. Then we have

$$
\begin{aligned}
\frac{d p_{\theta}}{d t} & =\mathfrak{B}_{1}(\mathfrak{b})\left(\ell^{2} m_{\text {rest }}+\frac{1}{4 \pi \alpha^{\prime}}\left(\pi T_{H}+\sqrt{\pi^{2} T_{H}^{2}-2 \ell^{2}}\right)\right) \frac{a^{2}}{2} \sin \left(2 \Theta_{0}\right)-\frac{\omega_{\theta} r_{H}^{2}}{2 \pi \alpha^{\prime}}+\ldots \\
\frac{d p_{\phi}}{d t} & =-\frac{1}{2 \pi \alpha^{\prime}}\left(\omega_{\phi} r_{H}^{2}-\mathfrak{p s i n}\left(\Theta_{0}\right)^{2} a\right)+\ldots \\
\frac{d p_{\psi}}{d t} & =-\frac{1}{2 \pi \alpha^{\prime}}\left(\omega_{\psi} r_{H}^{2}-\mathfrak{q} \sin \left(\Theta_{0}\right)^{2} a\right)+\ldots
\end{aligned}
$$

where $r_{H}$ is given by (A.4).

It is also instructive to obtain a relation to friction coefficients. One can only calculate this from the first terms of (3.25)-(3.27), related to the slow motion of the quark in a plasma. The second term in (3.25) can be centripetal force. So we find with (A.4)

$$
\mu=\left(\frac{\pi T}{\ell}\right)^{2} \frac{1}{2 m \pi \alpha^{\prime}}
$$

where $p=\frac{m \omega}{\ell^{2}}$.

\subsection{Energy of the static string in the 5d Kerr-AdS background}

The total energy of the string with the worldsheet parametrization $\left(\sigma^{0}, \sigma^{1}\right)=(t, r)$ is given by $(2.10)$, so

$$
E=-\frac{1}{2 \pi \alpha^{\prime}} \int d r \pi^{0}
$$

where by virtue of (2.4) the conjugate momentum for a string in (2.41) reads

$$
\pi_{t}^{0}=\sqrt{-g}\left(g^{t r} G_{t \phi} \phi^{\prime}+g^{t r} G_{t \psi} \psi^{\prime}+g^{t t} G_{t t}+g^{t t} G_{t \phi} \dot{\phi}+g^{t t} G_{t \psi} \dot{\psi}\right) .
$$

A single quark at rest is described by a static string solution with

$$
\theta(r, t)=\theta_{0}, \quad \phi(t, r)=\phi_{0}, \quad \psi(t, r)=\psi_{0},
$$

so eq. (3.30) with (3.2) is represented by

$$
\pi_{t}^{0}=g^{t t} G_{t t}=G_{r r} G_{t t} .
$$

Taking into account that for the 5d Kerr-AdS with one rotational parameter (2.41) we have

$$
G_{r r}=\frac{\rho^{2}}{\Delta_{r}}, \quad G_{t t}=-\frac{\Delta_{r}}{\rho^{2}}+a^{2} \frac{\Delta_{\theta} \sin ^{2} \theta}{\rho^{2}}
$$


where $\Delta_{\theta}$ and $\Delta_{r}$ are defined by (2.42). Plugging (3.33) into (3.32) we get

$$
\pi_{t}^{0}=-\left(1-a^{2} \frac{\Delta_{\theta}}{\Delta_{r}} \sin ^{2} \theta\right) .
$$

We note that comparing to [39] in (3.34) we have the second term related to a contribution with a rotational parameter $a^{2}, \Delta_{r}$, which root defines a location of the horizon. Therefore, there is a divergence related to a pole for $\Delta_{r}=0$. This can be regularized by introducing a cut-off. So to regularize this divergence we perform an integration from $r_{H}+\epsilon$. So for eq. (3.29) with (3.34) we have

$$
E=\frac{1}{2 \pi \alpha^{\prime}} \int_{r_{H}+\epsilon}^{r_{m}} d r\left(1-a^{2} \frac{\Delta_{\theta}}{\Delta_{r}} \sin ^{2} \theta\right)
$$

where $r_{m}$ is a quark location. We note that the relations (3.34)-(3.35) are exact and work for an arbitrary parameter $a$.

It's valuable to consider an expansion of (3.35) by order in small $a$. For (3.34) we get

$$
1-a^{2} \frac{\Delta_{\theta}}{\Delta_{r}} \sin ^{2} \theta \approx 1-a^{2} \frac{1}{r^{2}+\ell^{2} r^{4}-2 M} \sin ^{2} \theta
$$

Plugging (3.36) into (3.35) and integrating we get

$$
E=\left.\frac{1}{2 \pi \alpha^{\prime}}\left(r-\left(\frac{\tan ^{-1}\left(\frac{\sqrt{2} \ell r}{\sqrt{1-\sqrt{1+8 \ell^{2} M}}}\right)}{\sqrt{1-\sqrt{1+8 \ell^{2} M}}}-\frac{\tan ^{-1}\left(\frac{\sqrt{2} \ell r}{\sqrt{1+\sqrt{1+8 \ell^{2} M}}}\right)}{\sqrt{1+\sqrt{1+8 \ell^{2} M}}}\right) \frac{\sqrt{2} a^{2} \ell \sin ^{2} \theta}{\sqrt{1+8 \ell^{2} M}}\right)\right|_{r_{H}+\epsilon} ^{r_{m}} .
$$

The case of the zero temperature limit corresponds to $M=0$, so expanding (3.37) near $M=0$ we get

$$
\begin{aligned}
\left.E\right|_{T=0} & =\left.\frac{1}{2 \pi \alpha^{\prime}}\left(r-\left(-\frac{1}{\sqrt{2} \ell r}+\frac{i \pi}{4 \ell \sqrt{M}}-\frac{\tan ^{-1}(\ell r)}{\sqrt{2}}\right) \sqrt{2} a^{2} \ell \sin ^{2} \theta\right)\right|_{r_{H}+\epsilon} ^{r_{m}} \\
& =\frac{1}{2 \pi \alpha^{\prime}}\left(r_{m}+\left(\frac{1}{\ell r_{m}}+\tan ^{-1}\left(\ell r_{m}\right)-\frac{1}{\varepsilon}\right) a^{2} \ell \sin ^{2} \theta\right)
\end{aligned}
$$

where using the relation for the horizon (A.2) we have $r_{H}=0$ with $M=0$ and define $\varepsilon=\ell \epsilon$. From eq. (3.38) we see that the energy of the quark at rest in the zero temperature limit differs from that one [39] by the contribution from the rotational parameter and an extra term $\frac{1}{\varepsilon} a^{2} \ell \sin ^{2} \theta$ related to the renormalization.

At zero temperature the renormalized energy equals to the (Lagrangian) mass $m$ of the quark. For the case of the zero temperature limit from (3.39) we get

$$
\left.E_{r e n}\right|_{T=0}=\frac{1}{2 \pi \alpha^{\prime}}\left(r_{m}+\left(\frac{1}{\ell r_{m}}+\tan ^{-1}\left(\ell r_{m}\right)\right) a^{2} \ell \sin ^{2} \theta\right)=m .
$$

Increasing the temperature the relation for the energy takes the form

$$
E=\frac{1}{2 \pi \alpha^{\prime}}\left(m-r_{H}-\left(\frac{\sqrt{2} \tanh ^{-1}\left(\frac{r_{H}+\epsilon}{r_{H}}\right)}{r_{H}}+\frac{\tan ^{-1}\left(\frac{\ell\left(r_{H}+\epsilon\right)}{\sqrt{2\left(\ell^{2} r_{H}^{2}+1\right)}}\right)}{\sqrt{2} \sqrt{\ell^{2} r_{H}^{2}+1}}\right) \frac{a^{2} \ell \sin ^{2} \theta}{\left(2 \ell^{2} r_{H}^{2}+1\right)}\right),
$$


where we also take into account the relations for the horizon (A.2), (A.5)-(A.6). Following [39] we consider (3.35) as the static thermal mass $M_{\text {rest }}$

$$
M_{\text {rest }}=m-\Delta m(T, a),
$$

where

$$
\begin{aligned}
\Delta m(T, a)= & \frac{\sqrt{\lambda}}{2 \pi}\left(\frac{\pi T_{H}+\sqrt{\pi^{2} T_{H}^{2}-2 \ell^{2}}}{2 \ell^{2}}+\left(-\frac{\tanh ^{-1}\left(1+\frac{2 \ell^{2} \epsilon}{\pi T_{H}+\sqrt{\pi^{2} T_{H}^{2}-2 \ell^{2}}}\right)}{\pi T_{H}+\sqrt{\pi^{2} T_{H}^{2}-2 \ell^{2}}}\right.\right. \\
& \left.\left.+\frac{\sqrt{2} \tan ^{-1}\left(\frac{\left(\pi T_{H}+\sqrt{\pi^{2} T_{H}^{2}-2 \ell^{2}}+2 \epsilon \ell^{2}\right)}{\sqrt{2} \sqrt{\pi^{2} T_{H}^{2}+\ell^{2}+\pi T_{H} \sqrt{\pi^{2} T_{H}^{2}-2 \ell^{2}}}}\right)}{\sqrt{\pi^{2} T_{H}^{2}+\ell^{2}+\pi T_{H} \sqrt{\pi^{2} T_{H}^{2}-2 \ell^{2}}}}\right) \frac{\sqrt{2} a^{2} \ell^{4} \sin ^{2} \theta}{\pi^{2} T_{H}^{2}+\pi T_{H} \sqrt{\pi^{2} T_{H}^{2}-2 \ell^{2}}}\right),
\end{aligned}
$$

where $\lambda=1 / \alpha^{\prime 2}$. We are also able to write down thermal corrections to a quark mass without expansion for an arbitrary value of $a$. Integrating eq. (3.35) we obtain

$$
\begin{aligned}
E=\frac{1}{2 \pi \alpha^{\prime}}(r+ & \left(\frac{\tan ^{-1}\left(\frac{\sqrt{2} \ell r}{\sqrt{1+a^{2} \ell^{2}+\sqrt{\left(1-a^{2} \ell^{2}\right)^{2}+8 \ell^{2} M}}}\right)}{\sqrt{1+a^{2} \ell^{2}+\sqrt{\left(1-a^{2} \ell^{2}\right)^{2}+8 \ell^{2} M}}}\right) \\
& \left.\left.-\frac{\tan ^{-1}\left(\frac{\sqrt{2} \ell r}{\sqrt{1+a^{2} \ell^{2}-\sqrt{\left(1-a^{2} \ell^{2}\right)^{2}+8 \ell^{2} M}}}\right)}{\sqrt{1+a^{2} \ell^{2}-\sqrt{\left(1-a^{2} \ell^{2}\right)^{2}+8 \ell^{2} M}}}\right) \frac{\sqrt{2} a^{2} \ell \Delta_{\theta} \sin ^{2} \theta}{\sqrt{8 \ell^{2} M+\left(1-a^{2} \ell^{2}\right)^{2}}}\right)\left.\right|_{r_{+}+\epsilon} ^{r_{m}} .
\end{aligned}
$$

Taking into account the relation for the horizon (2.43) we get the relation for the energy at finite temperature takes the form

$$
\begin{aligned}
E=\frac{1}{2 \pi \alpha^{\prime}} & \left(r_{m}+\left(\tan ^{-1}\left(\ell r_{m}\right)-\tan ^{-1}\left(\frac{r_{m}}{a}\right) \frac{1}{a \ell}\right) \frac{a^{2} \ell \Delta_{\theta} \sin ^{2} \theta}{\left(1-a^{2} \ell^{2}\right)}\right. \\
& \left.-r_{+}-\left(\frac{\tan ^{-1}\left(\frac{\ell r_{+}}{\sqrt{\left(r_{+}^{2} \ell^{2}+1\right)}}\right)}{\left(1-a^{2} \ell^{2}+2 r_{+}^{2} \ell^{2}\right) \sqrt{\left(r_{+}^{2} \ell^{2}+1\right)}}+\frac{\sqrt{2} \tanh ^{-1}\left(\frac{\left(r_{+}+\epsilon\right)}{r_{+}}\right)}{\left(1-a^{2} \ell^{2}+2 r_{+}^{2} \ell^{2}\right) r_{+}}\right) a^{2} \ell \Delta_{\theta} \sin ^{2} \theta\right),
\end{aligned}
$$

or in terms of (3.42) we have

$$
\begin{gathered}
m=\frac{\sqrt{\lambda}}{2 \pi}\left(r_{m}+\left(\tan ^{-1}\left(\ell r_{m}\right)-\tan ^{-1}\left(\frac{r_{m}}{a}\right) \frac{1}{a \ell}\right) \frac{a^{2} \ell \Delta_{\theta} \sin ^{2} \theta}{\left(1-a^{2} \ell^{2}\right)}\right. \\
\Delta m(T, a)=r_{+}+\left(\frac{\tan ^{-1}\left(\frac{\ell r_{+}}{\sqrt{\left(r_{+}^{2} \ell^{2}+1\right)}}\right)}{\left(1-a^{2} \ell^{2}+2 r_{+}^{2} \ell^{2}\right) \sqrt{\left(r_{+}^{2} \ell^{2}+1\right)}}+\frac{\sqrt{2} \tanh ^{-1}\left(\frac{\left(r_{+}+\epsilon\right)}{r_{+}}\right)}{\left(1-a^{2} \ell^{2}+2 r_{+}^{2} \ell^{2}\right) r_{+}}\right) a^{2} \ell \Delta_{\theta} \sin ^{2} \theta
\end{gathered}
$$

where the horizon is related to the temperature through (2.36). It is interesting to note that without performing expansion by $a$ we do not meet with the divergence (3.39). This is related with non analiticity of r.h.s. of $(3.44)$ on $a$. The divergence in $\tanh ^{-1}\left(\frac{\left(r_{+}+\epsilon\right)}{r_{+}}\right)$ at $\epsilon \rightarrow 0$ is the same in two approaches. 


\section{Drag force from the $5 d$ Kerr-AdS metric with two equal rotational parameters}

\subsection{Static curved string in the Boyer-Lindquist coordinates}

Here we consider the drag force from the $5 \mathrm{~d}$ Kerr-AdS metric with two rotational parameters that are equal in magnitude $a=b(2.38)-(2.39) .{ }^{3}$ As for the one non-zero rotational parameter we are going to solve the linearized string equation of motion for small rotational parameters. For the string worldsheet coordinates we take $\left(\sigma^{0}, \sigma^{1}\right)=(t, r)$ and the embedding is characterized by (3.1) as for the case with one rotational parameter. The Nambu-Goto action calculated through the determinant of the induced metric of a string worldsheet looks like (2.1), where the components of the induced metric are

$$
\begin{aligned}
g_{t t} & =G_{t t}+G_{\theta \theta} \dot{\theta}^{2}+G_{\phi \phi} \dot{\phi}^{2}+G_{\psi \psi} \dot{\psi}^{2}+2\left(G_{t \phi} \dot{\phi}+G_{t \psi} \dot{\psi}+G_{\psi \phi} \dot{\psi} \dot{\phi}\right) \\
g_{t r} & =G_{\theta \theta} \dot{\theta} \theta^{\prime}+G_{\phi \phi} \dot{\phi} \phi^{\prime}+G_{\psi \psi} \dot{\psi} \psi^{\prime}+G_{t \phi} \phi^{\prime}+G_{t \psi} \psi^{\prime}+G_{\psi \phi} \dot{\psi} \phi^{\prime}+G_{\psi \phi} \psi^{\prime} \dot{\phi}, \\
g_{r r} & =G_{\theta \theta} \theta^{\prime 2}+G_{\phi \phi} \phi^{\prime 2}+G_{\psi \psi} \psi^{\prime 2}+G_{r r}+2 G_{\psi \phi} \psi^{\prime} \phi^{\prime}
\end{aligned}
$$

We expand the transversal variables $\phi, \theta$ and $\psi$ by small $a$ as we do this in the previous case (3.5)

$$
\begin{aligned}
\phi(t, r) & =\Phi_{0}+a \beta_{1} \ell^{2} t+a \beta_{1} \phi_{1}(r)+\mathcal{O}\left(a^{2}\right) \\
\psi(t, r) & =\Psi_{0}+a \beta_{2} \ell^{2} t+a \beta_{2} \psi_{1}(r)+\mathcal{O}\left(a^{2}\right), \\
\theta(r) & =\Theta_{0}+a^{2} \theta_{1}(r)+\mathcal{O}\left(a^{4}\right),
\end{aligned}
$$

where as in the previous section $\beta_{1}, \beta_{2}$ are some parameters with $\beta_{1}^{2}+\beta_{2}^{2}=1$.

Then we obtain the determinant of the induced metric as follows

$$
\begin{aligned}
-g=( & \frac{a^{2} \ell^{2} \rho^{2}}{\Xi^{2}}\left(\beta_{1}^{2} \sin ^{2} \theta \phi_{1}^{\prime}+\beta_{2}^{2} \cos ^{2} \theta \psi_{1}^{\prime}\right)+\frac{2 M a^{4} \ell^{2}}{\Xi^{2} \rho^{2}}\left(\beta_{1} \sin ^{2} \theta+\beta_{2} \cos ^{2} \theta\right)\left(\beta_{1} \sin ^{2} \theta \phi_{1}^{\prime}+\beta_{2} \cos ^{2} \theta \psi_{1}^{\prime}\right) \\
& \left.+\frac{a^{2}}{\Xi}\left(\rho^{2} \ell^{2}-\frac{2 M}{\rho^{2}}\right)\left(\beta_{1} \sin ^{2} \theta \phi_{1}^{\prime}+\beta_{2} \cos ^{2} \theta \psi_{1}^{\prime}\right)\right)^{2} \\
- & \left(-1-\rho^{2} l^{2}+\frac{2 M}{\rho^{2}}+\frac{a^{2} \ell^{4} \rho^{2}}{\Xi}\left(\beta_{1}^{2} \sin ^{2} \theta+\beta_{2}^{2} \cos ^{2} \theta\right)+\frac{2 a^{4} \ell^{4} M}{\Xi^{2} \rho^{2}}\left(\beta_{1} \sin ^{2} \theta+\beta_{2} \cos ^{2} \theta\right)^{2}\right. \\
& \left.+2 \frac{a^{2} \ell^{2}}{\Xi}\left(\rho^{2} l^{2}-\frac{2 M}{\rho^{2}}\right)\left(\beta_{1} \sin ^{2} \theta+\beta_{2} \cos ^{2} \theta\right)\right) \\
& \times\left(\frac{\rho^{2}}{\Delta_{r}}+\frac{a^{4} \rho^{2}}{\Delta_{\theta}} \theta_{1}^{\prime 2}+\frac{a^{2} \rho^{2}}{\Xi}\left(\beta_{1}^{2} \sin ^{2} \theta \phi_{1}^{\prime 2}+\beta_{2}^{2} \cos ^{2} \theta \psi_{1}^{\prime 2}\right)+\frac{2 M a^{4}}{\Xi^{2} \rho^{2}}\left(\beta_{1} \sin ^{2} \theta \phi_{1}^{\prime}+\beta_{2} \cos ^{2} \theta \psi_{1}^{\prime}\right)^{2}\right),
\end{aligned}
$$

where $\Delta_{r}, \Delta_{\theta}, \Xi, \rho$ are given by $(2.39)-(2.40)$.

The first integrals for $\phi_{1}$ and $\psi_{1}$ can be found from the Nambu-Goto action with (4.7), in lower order by $a$, and we can write down

$$
\phi_{1}(r)=\mathfrak{p} \int_{r_{+}}^{r} \frac{d \bar{r}}{\bar{r}^{4} h(\bar{r})}, \quad \psi_{1}(r)=\mathfrak{q} \int_{r_{+}}^{r} \frac{d \bar{r}}{\bar{r}^{4} h(\bar{r})},
$$

\footnotetext{
${ }^{3}$ See the SageMath notebook https://cocalc.com/share/8a0fbfc77f4422e8e29b66770873d3110ffd95a6/ Kerr-AdS-5D-string-a_eq_b.ipynb for details on the calculation.
} 
where we also use the conserved quantities $\mathfrak{p}, \mathfrak{q}$ and $h(r)$ is given by (3.8). The equation of motion for $\theta^{\prime}$, that follows from the Lagrangian with (4.7) after substitution (4.8), reads

$$
\begin{aligned}
\Upsilon^{\prime}+\frac{2\left(r+2 \ell^{2} r^{3}\right)}{r^{4} h} & \Upsilon+\frac{\ell^{4} r^{4}\left(\beta_{1}^{2}-\beta_{2}^{2}+2\left(\beta_{1}-\beta_{2}\right)\right)}{2 r^{8} h^{2}} \sin \left(2 \Theta_{0}\right) \\
& +\frac{-\beta_{1}^{2} \mathfrak{p}^{2}+\beta_{2}^{2} \mathfrak{q}^{2}-4 M \ell^{2}\left(\beta_{1}-\beta_{2}\right)}{2 r^{8} h^{2}} \sin \left(2 \Theta_{0}\right)=0,
\end{aligned}
$$

where we define $\Upsilon=\theta_{1}^{\prime}$.

Owing to (A.5)-(A.6) we can represent the solution to eq. (4.9) $\left(\theta_{1}^{\prime}\right)$ as follows ${ }^{4}$

$$
\begin{aligned}
\theta_{1}^{\prime}= & \frac{\tilde{C}_{1}}{r^{4} h}-\frac{\sin \left(2 \Theta_{0}\right)}{2 r^{4} h}\left(\beta_{1}^{2}-\beta_{2}^{2}+2\left(\beta_{1}-\beta_{2}\right)\right) \ell^{2} r \\
& +\frac{\sin \left(2 \Theta_{0}\right)}{2\left(2 \ell^{2} r_{H}^{2}+1\right) r^{4} h} \log \left(\frac{r+r_{H}}{r-r_{H}}\right) \frac{\ell^{4} r_{H}^{4}\left(\beta_{1}^{2}-\beta_{2}^{2}\right)-2\left(\beta_{1}-\beta_{2}\right) \ell^{2} r_{H}^{2}-\left(\beta_{1}^{2} \mathfrak{p}^{2}-\beta_{2}^{2} \mathfrak{q}^{2}\right)}{r_{H}} \\
& +\frac{\ell \sin \left(2 \Theta_{0}\right)}{2\left(2 \ell^{2} r_{H}^{2}+1\right) r^{4} h} \times \tan ^{-1}\left(\frac{\ell r}{\sqrt{\ell^{2} r_{H}^{2}+1}}\right) \\
& \times \frac{\left(\ell^{2} r_{H}^{2}+1\right)^{2}\left(\beta_{1}^{2}-\beta_{2}^{2}\right)+2\left(\beta_{1}-\beta_{2}\right)\left(r_{H}^{2} \ell^{2}+1\right)-\left(\beta_{1}^{2} \mathfrak{p}^{2}-\beta_{2}^{2} \mathfrak{q}^{2}\right)}{\sqrt{\ell^{2} r_{H}^{2}+1}}
\end{aligned}
$$

where $\tilde{C}_{1}$ is a constant. The corresponding conjugate momenta can be calculated using (2.4). On the boundary $r \rightarrow+\infty$ they take the following form

$$
\begin{aligned}
\pi_{\theta}^{r}= & \left(\frac{2 \tilde{C}_{1}}{\sin \left(2 \Theta_{0}\right)}-\left(\beta_{1}^{2}-\beta_{2}^{2}+2\left(\beta_{1}-\beta_{2}\right)\right) \ell^{2} r-\frac{\beta_{1}^{2}-\beta_{2}^{2}+2\left(\beta_{1}-\beta_{2}\right)}{r}\right. \\
& \left.+\frac{\pi \ell}{2\left(2 \ell^{2} r_{H}^{2}+1\right)} \frac{\left(\ell^{2} r_{H}^{2}+1\right)^{2}\left(\beta_{1}^{2}-\beta_{2}^{2}\right)+2\left(\beta_{1}-\beta_{2}\right)\left(r_{H}^{2} \ell^{2}+1\right)-\left(\beta_{1}^{2} \mathfrak{p}^{2}-\beta_{2}^{2} \mathfrak{q}^{2}\right)}{\sqrt{\ell^{2} r_{H}^{2}+1}}\right) \sin \left(2 \Theta_{0}\right) \frac{a^{2}}{2}, \\
\pi_{\phi}^{r}= & \mathfrak{p} \sin \left(\Theta_{0}\right)^{2} a+\mathcal{O}\left(a^{2}\right), \\
\pi_{\psi}^{r}= & \mathfrak{q} \cos \left(\Theta_{0}\right)^{2} a+\mathcal{O}\left(a^{2}\right) .
\end{aligned}
$$

Thanks to (2.9) the drag force can be found as

$$
\begin{aligned}
\frac{d p_{\theta}}{d t}= & \left(-\frac{2 \tilde{C}_{1}}{\sin \left(2 \Theta_{0}\right)}+\left(\beta_{1}^{2}-\beta_{2}^{2}+2\left(\beta_{1}-\beta_{2}\right)\right) \ell^{2} r+\frac{\beta_{1}^{2}-\beta_{2}^{2}+2\left(\beta_{1}-\beta_{2}\right)}{r}-\frac{\pi \ell}{2\left(2 \ell^{2} r_{H}^{2}+1\right)}\right. \\
& \left.\times \frac{\left(\ell^{2} r_{H}^{2}+1\right)^{2}\left(\beta_{1}^{2}-\beta_{2}^{2}\right)+2\left(\beta_{1}-\beta_{2}\right)\left(r_{H}^{2} \ell^{2}+1\right)-\left(\beta_{1}^{2} \mathfrak{p}^{2}-\beta_{2}^{2} \mathfrak{q}^{2}\right)}{\sqrt{\ell^{2} r_{H}^{2}+1}}\right) \frac{\sin \left(2 \Theta_{0}\right) a^{2}}{4 \pi \alpha^{\prime}} \\
\frac{d p_{\phi}}{d t}= & -\frac{1}{2 \pi \alpha^{\prime}} \mathfrak{p} \sin \left(\Theta_{0}\right)^{2} a+\mathcal{O}\left(a^{2}\right), \\
\frac{d p_{\psi}}{d t}= & -\frac{1}{2 \pi \alpha^{\prime}} \mathfrak{q} \cos \left(\Theta_{0}\right)^{2} a+\mathcal{O}\left(a^{2}\right) .
\end{aligned}
$$

As in the case with one non-zero rotational parameter, the component (4.14) has a divergent term with $r \rightarrow+\infty$ related to the infinite mass of the heavy quark

$$
\frac{d p_{\theta}}{d t}=\left(\mathfrak{B}_{2}(\mathfrak{b}) \ell^{2} r+\ldots\right) \frac{\sin \left(2 \Theta_{0}\right) a^{2}}{4 \pi \alpha^{\prime}},
$$

\footnotetext{
${ }^{4}$ The constant $\tilde{C}_{1}$ differs from $C_{1}$ by to including an imaginary constant.
} 
where as in the previous section we introduce a parameter $\mathfrak{B}_{2}(\mathfrak{b})=\beta_{1}^{2}-\beta_{2}^{2}+2\left(\beta_{1}-\beta_{2}\right)$ and we use parametrization $\beta_{1}=\sin \mathfrak{b}, \beta_{2}=\cos \mathfrak{b}$, so $\mathfrak{B}_{2}(\mathfrak{b})=-\cos (2 \mathfrak{b})+2(\sin (\mathfrak{b})-\cos (\mathfrak{b}))$. We see that it vanishes for the special choice of the parameters $\beta_{1}=\beta_{2}$.

For the special cases we have

- $\mathfrak{b}=\pi / 2, \mathfrak{B}_{2}(\mathfrak{b})=3$

$$
\begin{aligned}
\frac{d p_{\theta}}{d t} & =\left(-\frac{2 \tilde{C}_{1}}{\sin \left(2 \Theta_{0}\right)}+3 \ell^{2} r+\frac{3}{r}-\frac{\pi \ell}{2\left(2 \ell^{2} r_{H}^{2}+1\right)} \frac{\left(\ell^{2} r_{H}^{2}+2\right)^{2}-1-\mathfrak{p}^{2}}{\sqrt{r_{H}^{2} \ell^{2}+1}}\right) \frac{a^{2} \sin \left(2 \Theta_{0}\right)}{2}, \\
\frac{d p_{\phi}}{d t} & =-\frac{1}{2 \pi \alpha^{\prime}} \mathfrak{p} \sin \left(\Theta_{0}\right)^{2} a+\mathcal{O}\left(a^{2}\right), \quad \frac{d p_{\psi}}{d t}=0 .
\end{aligned}
$$

- $\mathfrak{b}=\pi / 4, \mathfrak{B}_{2}(\mathfrak{b})=0$

$$
\begin{aligned}
\frac{d p_{\theta}}{d t} & =\left(-\frac{2 \tilde{C}_{1}}{\sin \left(2 \Theta_{0}\right)}-\frac{\pi \ell}{4\left(2 \ell^{2} r_{H}^{2}+1\right)} \frac{\left(-\mathfrak{p}^{2}+\mathfrak{q}^{2}\right)}{\sqrt{r_{H}^{2} \ell^{2}+1}}\right) \sin \left(2 \Theta_{0}\right) \frac{a^{2}}{2}, \\
\frac{d p_{\phi}}{d t} & =-\frac{1}{2 \pi \alpha^{\prime}} \mathfrak{p} \sin \left(\Theta_{0}\right)^{2} a+\mathcal{O}\left(a^{2}\right), \\
\frac{d p_{\psi}}{d t} & =-\frac{1}{2 \pi \alpha^{\prime}} \mathfrak{q} \cos \left(\Theta_{0}\right)^{2} a+\mathcal{O}\left(a^{2}\right) .
\end{aligned}
$$

It should be noted that $\frac{d p_{\theta}}{d t}(4.20)$ disappears for $\mathfrak{p}=\mathfrak{q}$ and $\tilde{C}_{1}=0$.

\subsection{Static curved string in global AdS coordinates}

Now we are going to consider the string in the $5 \mathrm{~d}$ Kerr-AdS background with $a=b$ written in global AdS coordinates (2.45). ${ }^{5}$ For the worldsheet coordinates we set $\sigma^{0}=T, \sigma^{1}=y$. We suppose that the embedding is given by

$$
\Theta=\Theta(T, y), \quad \Phi=\Psi(T, y), \quad \Psi=\Psi(T, y) .
$$

The string action is given by (2.1), where the components of the induced metric (2.2) are

$$
\begin{aligned}
g_{T T} & =G_{T T}+G_{\Theta \Theta} \dot{\Theta}^{2}+G_{\Phi \Phi} \dot{\Phi}^{2}+G_{\Psi \Psi} \dot{\Psi}^{2}+2\left(G_{T \Phi} \dot{\Phi}+G_{T \Psi} \dot{\Psi}+G_{\Psi \Phi} \dot{\Psi} \dot{\Phi}\right) \\
g_{T y} & =G_{\Theta \Theta} \dot{\Theta} \Theta^{\prime}+G_{\Phi \Phi} \dot{\Phi} \Phi^{\prime}+G_{\Psi \Psi} \dot{\Psi} \Psi^{\prime}+G_{t \Phi} \Phi^{\prime}+G_{t \Psi} \Psi^{\prime}+G_{\Psi \Phi} \dot{\Psi} \Phi^{\prime}+G_{\Psi \Phi} \Psi^{\prime} \dot{\Phi} \\
g_{y y} & =G_{\Theta \Theta} \Theta^{\prime 2}+G_{\Phi \Phi} \Phi^{\prime 2}+G_{\Psi \Psi} \Psi^{\prime 2}+G_{y y}+2 G_{\Psi \Phi} \Psi^{\prime} \Phi^{\prime}
\end{aligned}
$$

We take the following expansion for the transversal coordinates

$$
\begin{aligned}
\Phi(T, y) & =\Phi_{0}+a \beta_{1} \ell^{2} T+a \beta_{1} \Phi_{1}(y)+\mathcal{O}\left(a^{2}\right), \\
\Theta(y) & =\Theta_{0}+a^{2} \Theta_{1}(y)+\mathcal{O}\left(a^{4}\right), \\
\Psi(T, y) & =\Psi_{0}+a \beta_{2} \ell^{2} T+a \beta_{2} \Psi_{1}(y)+\mathcal{O}\left(a^{2}\right) .
\end{aligned}
$$

\footnotetext{
${ }^{5}$ See the SageMath notebook https://cocalc.com/share/0577fb7f5f67a290a1c863b0eb5f069b6df747b2/ Kerr-AdS-5D-string-a_eq_b-AdS.ipynb for details on the calculation.
} 
Plugging (4.27)-(4.29) into (4.24)-(4.26) we calculate the determinant of the induced metric

$$
\begin{aligned}
|g|= & \left(\ell^{2} y^{2}\left(\beta_{1}^{2} \sin ^{2} \Theta \Phi_{1}^{\prime}+\beta_{2}^{2} \cos ^{2} \Theta \Psi_{1}^{\prime}\right)+\frac{2 \ell^{2} a^{2} M}{\Xi^{2} y^{2}}\left(\beta_{1}^{2} \sin ^{4} \Theta \Phi_{1}^{\prime}+\cos ^{4} \Theta \beta_{2}^{2} \Psi_{1}^{\prime}\right)\right. \\
& \left.-\frac{2 M}{y^{2} \Xi^{2}}\left(\sin ^{2} \Theta \beta_{1} \Phi_{1}^{\prime}+\cos ^{2} \Theta \beta_{2} \Psi_{1}^{\prime}\right)+\frac{2 M a^{2} \sin ^{2} \Theta \cos ^{2} \Theta}{\Xi^{2} y^{2}} \beta_{1} \beta_{2} \ell^{2}\left(\Phi_{1}^{\prime}+\Psi_{1}^{\prime}\right)\right)^{2} a^{4} \\
& -\left(-\left(1+y^{2} l^{2}-\frac{2 M}{y^{2} \Xi^{2}}\right)+\left(\beta_{1}^{2} \sin ^{2} \Theta+\beta_{2}^{2} \cos ^{2} \Theta\right) a^{2} y^{2} \ell^{4}\right. \\
& \left.+\left(\sin ^{2} \Theta \beta_{1}+\beta_{2} \cos ^{2} \Theta\right)^{2} \frac{2 a^{4} \ell^{4} M}{\Xi^{2} y^{2}}-4 \frac{a^{2} M \ell^{2}}{y^{2} \Xi^{2}}\left(\sin ^{2} \Theta \beta_{1}+\cos ^{2} \Theta \beta_{2}\right)\right) \\
& \times\left(y^{2} a^{4} \Theta_{1}^{\prime 2}+\frac{y^{4}}{y^{4}\left(1+y^{2} \ell^{2}\right)-\frac{2 M}{\Xi^{2}} y^{2}+\frac{2 M a^{2}}{\Xi^{3}}}\right. \\
& \left.+\left(\beta_{1}^{2} \Phi_{1}^{\prime 2} \sin ^{2} \Theta+\beta_{2}^{2} \Psi_{1}^{\prime 2} \cos ^{2} \Theta\right) a^{2} y^{2}+\frac{2 a^{4} M}{\Xi^{2} y^{2}}\left(\sin ^{2} \Theta \beta_{1} \Phi_{1}^{\prime}+\cos ^{2} \Theta \beta_{2} \Psi_{1}^{\prime}\right)^{2}\right),
\end{aligned}
$$

with $\Xi$ given by (2.40).

As the in Boyer-Lindquist coordinates the variables $\Phi_{1}$ and $\Psi_{1}$ are cyclic, so we can find the first integrals for $\Phi_{1}$ and $\Psi_{1}$ for the Nambu-Goto action (2.1) with (4.30)

$$
\Phi_{1}(y)=\mathfrak{p} \int_{y_{+}}^{y} \frac{d \bar{y}}{\bar{y}^{2}+\ell^{2} \bar{y}^{4}-2 M}, \quad \Psi_{1}(y)=\mathfrak{q} \int_{y_{+}}^{y} \frac{d \bar{y}}{\bar{y}^{2}+\ell^{2} \bar{y}^{4}-2 M}
$$

Substituting (4.31) into (4.30) we derive the equation of motion for $\Theta_{1}$

$$
\Upsilon^{\prime}+\frac{2\left(y+2 \ell^{2} y^{3}\right)}{-2 M+y^{2}+\ell^{2} y^{4}} \Upsilon_{1}+\frac{-\beta_{1}^{2} \mathfrak{p}^{2}+\beta_{2}^{2} \mathfrak{q}^{2}-4\left(\beta_{1}-\beta_{2}\right) \ell^{2} M+\ell^{4} y^{4}\left(\beta_{1}^{2}-\beta_{2}^{2}\right)}{2\left(-2 M+y^{2}+\ell^{2} y^{4}\right)^{2}} \sin \left(2 \Theta_{0}\right)=0
$$

with $\Upsilon=\Theta_{1}^{\prime}$. The solution to (4.32) leads to the following relation

$$
\begin{aligned}
\Theta_{1}^{\prime}= & \frac{\tilde{C}_{1}}{y^{4} \ell^{2}+y^{2}-2 M} \\
& +\frac{-2 \ell^{2} y_{H}^{2}\left(1+\ell^{2} y_{H}^{2}\right)\left(\beta_{1}-\beta_{2}\right)+\left(\beta_{1}^{2}-\beta_{2}^{2}\right) \ell^{4} y_{H}^{4}-\beta_{1}^{2} \mathfrak{p}^{2}+\beta_{2}^{2} \mathfrak{q}^{2}}{\left(2 \ell^{2} y_{H}^{2}+1\right) \ell y_{H}} \\
& \times \frac{\ell}{2\left(y^{4} \ell^{2}+y^{2}-2 M\right)} \tanh ^{-1}\left(\frac{y}{y_{H}}\right) \sin \left(2 \Theta_{0}\right) \\
& +\frac{-2 \ell^{2} y_{H}^{2}\left(1+\ell^{2} y_{H}^{2}\right)\left(\beta_{1}-\beta_{2}\right)+\left(\beta_{1}^{2}-\beta_{2}^{2}\right)\left(\ell^{2} y_{H}^{2}+1\right)^{2}-\beta_{1}^{2} \mathfrak{p}^{2}+\beta_{2}^{2} \mathfrak{q}^{2}}{\left(2 \ell^{2} y_{H}^{2}+1\right) \sqrt{\ell^{2} y_{H}^{2}+1}} \\
& \times \frac{\ell}{2\left(y^{4} \ell^{2}+y^{2}-2 M\right)} \tan ^{-1}\left(\frac{\ell y}{\sqrt{\ell^{2} y_{H}^{2}+1}}\right) \sin \left(2 \Theta_{0}\right)-\frac{\left(\beta_{1}^{2}-\beta_{2}^{2}\right) \ell^{2} y \sin \left(2 \Theta_{0}\right)}{2\left(-2 M+y^{2}+\ell^{2} y^{4}\right)},
\end{aligned}
$$

where $\tilde{C}_{1}$ is a constant of integration. Using (2.4), (4.31), (4.33) and taking into ac- 
count (2.9) we can write down the drag force components

$$
\begin{aligned}
\frac{d p_{\theta}}{d t}= & \left(-\frac{2 \tilde{C}_{1}}{\sin \left(2 \Theta_{0}\right)}+\left(\beta_{1}^{2}-\beta_{2}^{2}\right) \ell^{2} y+\frac{\beta_{1}^{2}-\beta_{2}^{2}}{y}-\frac{\pi \ell}{2\left(2 \ell^{2} y_{H}^{2}+1\right)}\right. \\
& \left.\times \frac{\left(-2 \ell^{2} y_{H}^{2}\left(1+\ell^{2} y_{H}^{2}\right)\left(\beta_{1}-\beta_{2}\right)+\left(\beta_{1}^{2}-\beta_{2}^{2}\right)\left(\ell^{2} y_{H}^{2}+1\right)^{2}-\beta_{1}^{2} \mathfrak{p}^{2}+\beta_{2}^{2} \mathfrak{q}^{2}\right)}{\sqrt{\ell^{2} y_{H}^{2}+1}}\right) \frac{\sin \left(2 \Theta_{0}\right) a^{2}}{4 \pi \alpha^{\prime}} \\
\frac{d p_{\phi}}{d t}= & -\frac{1}{2 \pi \alpha^{\prime}} \mathfrak{p} \sin \left(\Theta_{0}\right)^{2} a+\mathcal{O}\left(a^{2}\right), \\
\frac{d p_{\psi}}{d t}= & -\frac{1}{2 \pi \alpha^{\prime}} \mathfrak{q} \cos \left(\Theta_{0}\right)^{2} a+\mathcal{O}\left(a^{2}\right) .
\end{aligned}
$$

Comparing the result with this for two rotational parameter $a=b$ performed in BoyerLindquist coordinates, we find that the relations for $\frac{d p_{\theta}}{d t}$ have the common dependence on the radial coordinates ( $y$ and $r$, correspondingly) and the horizons $\left(y_{H}, r_{H}\right)$. At the same time the coefficients for these terms are different for the Boyer-Lindquist and AdS coordinates (we have $\beta_{1}^{2}-\beta_{2}^{2}+2\left(\beta_{1}-\beta_{2}\right)$ and $\beta_{1}^{2}-\beta_{2}^{2}$, correspondingly). This can be associated to that the first coordinate system is related to a rotating-at-infinity frame, while the second one corresponds to a static-at-infinity frame. However, one can reach an exactly same answer if we choose the parameters for the string dynamics as $\sin \mathfrak{b}=\beta_{1} \cos \mathfrak{b}=\beta_{2}$ and take $\mathfrak{b}=\frac{\pi}{4}$. Therefore, we have

$$
\begin{aligned}
\frac{d p_{\theta}}{d t} & =\left(-\frac{2 \tilde{C}_{1}}{\sin \left(2 \Theta_{0}\right)}-\frac{\pi \ell}{4\left(2 \ell^{2} y_{H}^{2}+1\right)} \frac{\left(-\mathfrak{p}^{2}+\mathfrak{q}^{2}\right)}{\sqrt{\ell^{2} y_{H}^{2}+1}}\right) \frac{\sin \left(2 \Theta_{0}\right) a^{2}}{4 \pi \alpha^{\prime}}, \\
\frac{d p_{\phi}}{d t} & =-\frac{1}{2 \pi \alpha^{\prime}} \mathfrak{p} \sin \left(\Theta_{0}\right)^{2} a+\mathcal{O}\left(a^{2}\right) \\
\frac{d p_{\psi}}{d t} & =-\frac{1}{2 \pi \alpha^{\prime}} \mathfrak{q} \cos \left(\Theta_{0}\right)^{2} a+\mathcal{O}\left(a^{2}\right),
\end{aligned}
$$

that matches with (4.20)-(4.22). Moreover, we can also observe a degenerate case if the parameters $\mathfrak{p}=\mathfrak{q}$ and the constant $\tilde{C}_{1}=0$. Then the component (4.37) of the drag force just equals to zero. This is in agreement with the calculations in the Boyer-Lindquist coordinates and with the hydrodynamical results from section 2.2 eq. (2.24) where we consider the case of equal angular velocities $\Omega_{a}=\Omega_{b}$.

\section{Conclusion and discussion}

In this paper we have studied the drag force acting on a heavy quark moving in the rotating quark gluon plasma within the context of holographic duality. We have considered a 5d Kerr-AdS black hole as a holographic dual of a 4d strongly coupled rotating QGP. We have focused on cases where the black hole solution has either only one non-zero rotational parameter $(a \neq 0, b=0)$ or two rotational parameters that are equal $(a=b \neq$ $0)$. These cases are related to the presence of one and two Casimir invariants for $\mathrm{SO}(4)$, correspondingly.

Following the holographic prescription we have associated the heavy quark with an end of a string suspended on the boundary of the Kerr-AdS black hole into its interior. 
We note that in this work the ansatz under consideration corresponds to the fixed quark in a rotating medium. We focused on the case when the rotational parameter is small. We have solved the string equations of motion order by order in $a$. Then we have found the corresponding conjugate momenta, which are related to components of the drag force.

In the case of a single rotational parameter we have performed calculations in BoyerLindquist coordinates. We have established that the relation for the leading term of the drag force is in agreement with the prediction of the work [32], which considered a lower dimensional holographic duality, namely, a 4d Kerr-AdS black hole for a 3d rotating quarkgluon plasma. This is actually not surprising, since $4 \mathrm{~d}$ rotating black holes have just one Casimir invariant corresponding to $\mathrm{SO}(3)$. We have also found corrections to the thermal quark mass at rest. It worth to be noted at zero temperature limit that the cut-off term has a contribution from rotation comparing to the result [39].

In the case of Kerr-AdS black hole with two equal rotational parameters we have calculated the drag force both in Boyer-Lindquist and in global AdS ones. We have observed that the results for the drag forces have the same dependence on the radial coordinates, however the coefficients may be different. This happens because the Boyer-Lindquist coordinates are related to a rotating frame, while the AdS coordinates - to a non-rotating frame. We have seen that there is a degenerated case when the drag force vanishes for certain values of the coefficients. This result matches with calculations from the hydrodynamical approach on 4-dimensional sphere.

To summarize our considerations about applied holography for Kerr- $A d S_{5}$ we can mention that

- rotation has some influence on the fist order phase transition,

- it decreases the critical temperature,

- the critical end point depends on some combination of two rotational parameters.

- The drag force contains two terms; one of them is related the slow motion of the quark with respect to the fluid, the second one is interpreted as a centripetal force.

- The friction coefficient depends on the temperature quadratically,

- the centripetal term depends on the temperature linearly,

- the centripetal force vanishes in the case of two equal rotational parameter.

- Comparing to [39] the energy of the quark at rest in the zero temperature limit has a contribution from the rotational parameter.

These phenomena can lead to measurable experimental signals in heavy-ion collisions. Besides the transport properties, rotation can affect on the phase structure and phase transition of matter at energies relevant to LHC and RHIC.

A straightforward problem for future study is to investigate the drag force in the $5 \mathrm{~d}$ Kerr-AdS black hole with two non-equal rotational parameters and to trace the influence of the second rotational parameter on energy loss. Our consideration can be also generalized to higher dimensional cases [65] with a compactification (see also [66]), as well for more 
complicate string configurations [45]. Also it would be interesting to apply exact results of [67] to the problem treated here.

Another interesting problem for some future work is the study of the drag forces in the rotating charged Kerr-AdS metric [68], which describes a conformal plasma with non-zero chemical potential. In this case the phase diagram for $a=b$ in the space $(T, a, q)$ is very similar to the phase diagram presented in figure 4 for two rotational parameters.

Note that the Kerr-AdS black hole gives a good description of QGP at extremely high temperature, where the system tends to the conformal limit. Such a temperature is relevant to LHC and RHIC energies. Hoverer at low temperature, typical for FAIR and NICA, the conformal symmetry is broken and non-conformal holographic models are more relevant [69]. The effects of vorticity in peripheral collisions at the NICA facility are widely discussed and it is expected to have hyperon polarization at NICA energies [70-75]. Therefore, to describe holographically hyperon polarization at NICA energies we have to deal with 5 d deformed metrics with rotation. To date, only one parametric deformed KerrAdS solution is known (a solution with one rotational parameter) [76-79]. In fact from the side of experimental data, it is not obvious that two parameters should be introduced to describe rotated QGP produced in HIC. But what is for sure, is that the deformation of Kerr-AdS solutions which would be relevant in the context of NICA, has to include the electromagnetic field. From one side, this is because here we deal with non-zero chemical potential and within the holography it is described by temporal component of the Maxwell field. From the other side, a huge magnetic field is also expected [80] to be there. Study of perturbations of the electromagnetic field in deformed rotating metric is also relevant in the context of the direct photons [69].

We would like to mention that the properties of rotating strongly interacting matter were also studied with lattice QCD [81]. As compared with chemical potential, rotation is a simpler task for lattice studies. It is also predicted a dependence of transport phenomena on rotation, [82-84] and this is a subject of future investigation to find closer relation with our calculation. This is especially interesting since lattice calculations can be done for two non-zero parameters of rotation and we are going to compare them with our future studies.

\section{Acknowledgments}

The authors are grateful to Nata Atmaja for useful clarifications. The authors also thank for clarifying discussions Hristo Dimov, Oleg Teryaev and Sasha Zhiboedov. The work of IA was performed at the Steklov International Mathematical Center and supported by the Ministry of Science and Higher Education of the Russian Federation (agreement no. 07515-2019-1614). AG is grateful to the CERN Theory Division and the Paris Observatory, LUTh for kind hospitality, where a part of this work was done. The work of IA and AG is supported by Russian Foundation for Basic Research (RFBR) grant No. 18-02-40069 mega. IA is also grateful for support provided "BASIS" Science Foundation (grant No. 18-1-1-80-4). AG is also supported by the JINR grant for young scientists No. 20-302-02 and the Landau-Heisenberg program. EG acknowledges support from CNRS 80 PRIME program TNENGRAV. 


\section{A Some facts on Kerr-AdS black holes}

\section{A.1 Special cases of 5d Kerr-AdS black holes}

Taking $a=b=0$ in (2.33) for Kerr-AdS metric we come to a Schwartzchild-AdS black hole

$$
d s^{2}=-\frac{r^{2}+r^{4} \ell^{2}-2 M}{r^{2}} d t^{2}+\frac{r^{2}}{r^{2}+r^{4} \ell^{2}-2 M} d r^{2}+r^{2} d \Omega_{3}^{2},
$$

where the horizon is defined by

$$
r_{H}=\frac{\sqrt{\sqrt{1+8 \ell^{2} M}-1}}{\sqrt{2} \ell} .
$$

The Hawking temperature is given by

$$
T_{H}=\frac{4 r_{H}^{2} \ell^{2}+2}{4 \pi r_{H}}
$$

so the location of the horizon is defined through the temperature as

$$
r_{H}=\frac{1}{2 \ell^{2}}\left(\pi T_{H}+\sqrt{\pi^{2} T_{H}^{2}-2 \ell^{2}}\right) .
$$

From (A.2) one can write down some useful relations

$$
\begin{aligned}
2 \ell^{2} r_{H}^{2} & =\sqrt{1+8 \ell^{2} M}-1, & 2 \ell^{2} r_{H}^{2}+1 & =\sqrt{1+8 \ell^{2} M}, \\
2 \ell^{4} r_{H}^{4} & =1+4 \ell^{2} M-\sqrt{1+8 \ell^{2} M}, & 4\left(\ell^{2} r_{H}^{2}+1\right)^{2} & =\left(\sqrt{1+8 \ell^{2} M}+1\right)^{2} .
\end{aligned}
$$

In the so-called AdS coordinates (2.45) the 5d Kerr-AdS solutions with $M=0$ come to the following form

$$
d s_{g A d S}^{2}=-\left(1+y^{2} \ell^{2}\right) d T^{2}+y^{2}\left(d \Theta^{2}+\sin ^{2} \Theta d \Phi^{2}+\cos ^{2} \Theta d \Psi^{2}\right)+\frac{d y^{2}}{\left(1+y^{2} \ell^{2}\right)},
$$

that is a well known form of the global representation of the AdS solution, often called static coordinates.

\section{A.2 Boundaries}

The metric on the boundary for (2.33) is

$$
d s_{\mathrm{BL}}^{2}=-d t^{2}+\frac{2 a \sin ^{2} \theta}{\Xi_{a}} d t d \phi+\frac{2 b \cos ^{2} \theta}{\Xi_{b}} d t d \psi+\frac{\ell^{2}}{\Delta_{\theta}} d \theta^{2}+\frac{\ell^{2} \sin ^{2} \theta}{\Xi_{a}} d \phi^{2}+\frac{\ell^{2} \cos ^{2} \theta}{\Xi_{b}} d \psi^{2} .
$$

From (A.7) it is easy to see that the $4 \mathrm{~d}$ conformal boundary of $5 \mathrm{~d}$ Kerr-AdS black hole is $4 \mathrm{~d} \mathbb{R} \times \mathbb{S}^{3}[26,29]$, which is reached with $y \rightarrow \infty$ :

$$
d s^{2}=-\ell^{2} d T^{2}+d \Theta^{2}+\sin ^{2} \Theta d \Phi^{2}+\cos ^{2} \Theta d \Psi^{2} .
$$

The boundary metrics (A.8) and (A.9) are related by

$$
d s_{\mathrm{BL}, \mathrm{bnd}}^{2}=\frac{y^{2}}{r^{2}} d s_{\text {gAdS,bnd }}^{2} \text {. }
$$




\section{B Strings in a 5d Kerr-AdS black hole}

\section{B.1 Straight string solution in global AdS}

Let us consider a string motion in the 5d Kerr-AdS background written in the AdS coordinates. The general form of the metric in these coordinates is complicated. However, under the assumption $M=0$ that corresponds to absence of the quark-gluon plasma the Kerr-AdS metric comes to the global AdS solution (A.7), that can be represented as

$$
d s^{2}=-y^{2} h(y) d T^{2}+y^{2}\left(d \Theta^{2}+\sin ^{2} \Theta d \Phi^{2}+\cos ^{2} \Theta d \Psi^{2}\right)+\frac{d y^{2}}{y^{2} h(y)},
$$

where

$$
h(y)=\ell^{2}+\frac{1}{y^{2}}
$$

We use the physical gauge with

$$
\left(\sigma^{0}, \sigma^{1}\right)=(T, y)
$$

For the embedding we have $X^{\mu}=X^{\mu}(\sigma)$, so

$$
\Theta=\Theta(T, y), \quad \Phi=\Phi(T, y), \quad \Psi=\Psi(T, y) .
$$

The induced metric is

$$
g_{\alpha \beta}=\left[\begin{array}{cc}
-y^{2} h+G_{I J} \dot{X}^{I} \dot{X}^{J} & G_{I J} \dot{X}^{\prime}{ }^{I} X^{\prime} J \\
G_{I J} \dot{X}^{\prime}{ }^{I} X^{\prime} J & \frac{1}{y^{2} h}+G_{I J} X^{\prime I} X^{\prime} J
\end{array}\right],
$$

where the indices run as $I, J=(\Theta, \Phi, \Psi)$ and ${ }^{\cdot}=\frac{d}{d T},{ }^{\prime}=\frac{d}{d y}$.

The Nambo-Goto action reads

$$
S_{N G}=\int d T d y \sqrt{|g|}
$$

with the determinant of the induced metric:

$$
\begin{aligned}
g= & -1+h^{-1}\left(\dot{\Theta}^{2}+\dot{\Phi}^{2} \sin ^{2} \Theta+\cos ^{2} \Theta \dot{\Psi}^{2}\right)-y^{4} h\left(\Theta^{\prime 2}+\Phi^{\prime 2} \sin ^{2} \Theta+\cos ^{2} \Theta \Psi^{\prime 2}\right) \\
& +y^{4}\left(\left(\dot{\Theta}^{2}+\dot{\Phi}^{2} \sin ^{2} \Theta+\cos ^{2} \Theta \dot{\Psi}^{2}\right)\left(\Theta^{\prime 2}+\Phi^{\prime 2} \sin ^{2} \Theta+\cos ^{2} \Theta \Psi^{\prime 2}\right)\right. \\
& \left.-\left(\dot{\Theta} \Theta^{\prime}+\sin ^{2} \Theta \dot{\Phi} \Phi^{\prime}+\cos ^{2} \Theta \dot{\Psi} \Psi^{\prime}\right)^{2}\right)
\end{aligned}
$$

assuming that fluctuations $\dot{X}^{I}, X^{\prime}$ are small we can write

$$
\sqrt{-g} \approx \sqrt{1-h^{-1}\left(\dot{\Theta}^{2}+\dot{\Phi}^{2} \sin ^{2} \Theta+\cos ^{2} \Theta \dot{\Psi}^{2}\right)+y^{4} h\left(\Theta^{\prime 2}+\Phi^{\prime 2} \sin ^{2} \Theta+\cos ^{2} \Theta \Psi^{\prime 2}\right)} .
$$

The equations of motion that follow from (B.6) with (B.8) take the following form

$$
\begin{aligned}
& \frac{\partial}{\partial y}\left(h y^{4} \frac{\Theta^{\prime}}{\sqrt{-g}}\right)-\frac{1}{h}\left(\frac{\partial}{\partial t} \frac{\dot{\Theta}}{\sqrt{-g}}\right)=\frac{\sin (2 \Theta)}{2 \sqrt{-g}}\left(h y^{4}\left(\Phi^{\prime 2}-\Psi^{\prime 2}\right)-\frac{\dot{\Phi}^{2}-\dot{\Psi}^{2}}{h}\right), \\
& \frac{\partial}{\partial y}\left(h y^{4} \frac{\Phi^{\prime}}{\sqrt{-g}}\right)-\frac{1}{h}\left(\frac{\partial}{\partial t} \frac{\dot{\Phi}}{\sqrt{-g}}\right)=0 \\
& \frac{\partial}{\partial y}\left(h y^{4} \frac{\Psi^{\prime}}{\sqrt{-g}}\right)-\frac{1}{h}\left(\frac{\partial}{\partial t} \frac{\dot{\Psi}}{\sqrt{-g}}\right)=0 .
\end{aligned}
$$


Eqs. (B.9)-(B.11) posses the static string solution that reads as

$$
\Theta=\Theta_{0}, \quad \Phi=\Phi_{0}, \quad \Psi=\Psi_{0},
$$

where $\Theta_{0}, \Phi_{0}$ and $\Psi_{0}$ are some constants corresponding to a massive quark at rest. We note that plugging the solution for the straight static string (B.12) into (B.7), we see that $-g$ is not positively defined. This fact was mentioned in [39].

The corresponding "time-dependent" solution in the Boyer-Lindquist coordinates with (2.44) is thus

$$
\psi=\Psi_{0} \quad \phi=\Phi_{0}-a \ell^{2} t, \quad \theta=\arccos \left(\frac{y}{r} \cos \Theta_{0}\right),
$$

where

$$
y^{2}=\frac{r^{2}\left(r^{2}+a^{2}\right)}{\left(1-a^{2} l^{2} \sin ^{2} \Theta_{0}\right) r^{2}+a^{2} \cos ^{2} \Theta_{0}} .
$$

\section{B.2 Curved string in 5d Kerr-AdS with one rotational parameter (supple- mentary relations)}

The determinant of the induced metric built on (3.2)-(3.4) is

$$
\begin{aligned}
-g= & \left(\left(a \Delta_{r}-a\left(r^{2}+a^{2}\right) \Delta_{\theta}\right) \frac{\sin ^{2} \theta}{\Xi_{a} \rho^{2}} \phi^{\prime}+\frac{\rho^{2}}{\Delta_{\theta}} \dot{\theta} \theta^{\prime}\right. \\
& \left.+\left(\Delta_{\theta}\left(r^{2}+a^{2}\right)^{2}-a^{2} \Delta_{r} \sin ^{2} \theta\right) \frac{\sin ^{2} \theta}{\Xi_{a}^{2} \rho^{2}} \dot{\phi} \phi^{\prime}+r^{2} \cos ^{2} \theta \dot{\psi} \psi^{\prime}\right)^{2} \\
- & \left(\frac{\rho^{2}}{\Delta_{r}}+\frac{\rho^{2}}{\Delta_{\theta}} \theta^{\prime 2}+\left(\Delta_{\theta}\left(r^{2}+a^{2}\right)^{2}-a^{2} \Delta_{r} \sin ^{2} \theta\right) \frac{\sin ^{2} \theta}{\Xi_{a}^{2} \rho^{2}} \phi^{\prime 2}+r^{2} \cos ^{2} \theta \psi^{\prime 2}\right) \\
& \times\left(\left(a^{2} \Delta_{\theta} \sin ^{2} \theta-\Delta_{r}\right) \frac{1}{\rho^{2}}+\left(a \Delta_{r}-a\left(r^{2}+a^{2}\right) \Delta_{\theta}\right) \frac{2 \sin ^{2} \theta}{\Xi_{a} \rho^{2}} \dot{\phi}\right. \\
& \left.\quad+\left(\Delta_{\theta}\left(r^{2}+a^{2}\right)^{2}-a^{2} \Delta_{r} \sin ^{2} \theta\right) \frac{\sin ^{2} \theta}{\Xi_{a}^{2} \rho^{2}} \dot{\phi}^{2}+\frac{\rho^{2}}{\Delta_{\theta}} \dot{\theta}^{2}+r^{2} \cos ^{2} \theta \dot{\psi}^{2}\right),
\end{aligned}
$$

where $\Delta_{r}, \Delta_{\theta}, \Xi_{a}, \rho$ are defined as (2.42).

Owing to (3.5) the components of the induced metric can be represented in the following way

$$
\begin{aligned}
g_{r t}= & \frac{a^{2} \sin ^{2} \theta}{\rho^{2} \Xi_{a}^{2}}\left(\beta_{1} \Xi_{a}\left(\Delta_{r}-\Delta_{\theta}\left(r^{2}+a^{2}\right)\right)+\beta_{1}^{2} \ell^{2}\left(\Delta_{\theta}\left(r^{2}+a^{2}\right)^{2}-a^{2} \Delta_{r} \sin ^{2} \theta\right)\right) \phi_{1}^{\prime} \\
& +a^{2} \ell^{2} \beta_{2}^{2} r^{2} \cos ^{2} \theta \psi_{1}^{\prime} \\
g_{t t}= & \frac{a^{2} \Delta_{\theta} \sin ^{2} \theta-\Delta_{r}}{\rho^{2}}+\frac{2 \beta_{1} a^{2} \ell^{2} \sin ^{2} \theta\left(\Delta_{r}-\Delta_{\theta}\left(r^{2}+a^{2}\right)\right)}{\rho^{2} \Xi_{a}} \\
& +\beta_{1}^{2} \frac{a^{2} \ell^{4} \sin ^{2} \theta}{\rho^{2} \Xi_{a}^{2}}\left(\Delta_{\theta}\left(r^{2}+a^{2}\right)^{2}-a^{2} \Delta_{r} \sin ^{2} \theta\right)+\beta_{2}^{2} a^{2} \ell^{4} r^{2} \cos ^{2} \theta \\
g_{r r}= & \frac{\rho^{2}}{\Delta_{r}}+\frac{a^{2} \beta_{1}^{2} \sin ^{2} \theta}{\Xi_{a}^{2} \rho^{2}}\left(\Delta_{\theta}\left(r^{2}+a^{2}\right)^{2}-\Delta_{r} a^{2} \sin ^{2} \theta\right) \phi_{1}^{\prime 2}+a^{4} \frac{\rho^{2}}{\Delta_{\theta}} \theta_{1}^{\prime 2} \\
& +\beta_{2}^{2} a^{2} r^{2} \cos ^{2} \theta \psi_{1}^{\prime 2} .
\end{aligned}
$$


Open Access. This article is distributed under the terms of the Creative Commons Attribution License (CC-BY 4.0), which permits any use, distribution and reproduction in any medium, provided the original author(s) and source are credited.

\section{References}

[1] L.D. Landau, On the multiparticle production in high-energy collisions, Izv. Akad. Nauk Ser. Fiz. 17 (1953) 51 [INSPIRE].

[2] J.D. Bjorken, Highly relativistic nucleus-nucleus collisions: the central rapidity region, Phys. Rev. D 27 (1983) 140 [INSPIRE].

[3] S.S. Gubser, Symmetry constraints on generalizations of Bjorken flow, Phys. Rev. D 82 (2010) 085027 [arXiv: 1006.0006] [inSPIRE].

[4] Z.-T. Liang and X.-N. Wang, Globally polarized quark-gluon plasma in non-central $A+A$ collisions, Phys. Rev. Lett. 94 (2005) 102301 [Erratum ibid. 96 (2006) 039901] [nucl-th/0410079] [INSPIRE].

[5] F. Becattini, F. Piccinini and J. Rizzo, Angular momentum conservation in heavy ion collisions at very high energy, Phys. Rev. C 77 (2008) 024906 [arXiv:0711.1253] [INSPIRE].

[6] Y. Jiang, Z.-W. Lin and J. Liao, Rotating quark-gluon plasma in relativistic heavy ion collisions, Phys. Rev. C 94 (2016) 044910 [Erratum ibid. 95 (2017) 049904] [arXiv: 1602.06580] [INSPIRE].

[7] W.-T. Deng and X.-G. Huang, Vorticity in heavy-ion collisions, Phys. Rev. C 93 (2016) 064907 [arXiv: 1603.06117] [INSPIRE].

[8] L.-G. Pang, H. Petersen, Q. Wang and X.-N. Wang, Vortical fluid and $\Lambda$ spin correlations in high-energy heavy-ion collisions, Phys. Rev. Lett. 117 (2016) 192301 [arXiv:1605.04024] [INSPIRE].

[9] STAR collaboration, Global $\Lambda$ hyperon polarization in nuclear collisions: evidence for the most vortical fluid, Nature 548 (2017) 62 [arXiv:1701.06657] [INSPIRE].

[10] STAR collaboration, Global polarization of $\Lambda$ hyperons in Au+Au collisions at $\sqrt{s_{N N}}=200 \mathrm{GeV}$, Phys. Rev. C 98 (2018) 014910 [arXiv: 1805.04400] [INSPIRE].

[11] STAR collaboration, Global and local polarization of $\Lambda$ hyperons in Au+Au collisions at $200 \mathrm{GeV}$ from STAR, Nucl. Phys. A 982 (2019) 511 [arXiv:1808.10482] [inSPIRE].

[12] STAR collaboration, Highlights from the STAR experiment, Nucl. Phys. A 982 (2019) 29 [arXiv: 1809.05713] [INSPIRE].

[13] Y. Jiang and J. Liao, Pairing phase transitions of matter under rotation, Phys. Rev. Lett. 117 (2016) 192302 [arXiv:1606.03808] [INSPIRE].

[14] S. Dey, S. De and B.R. Majhi, Gravity dual of Navier-Stokes equation in a rotating frame through parallel transport, Phys. Rev. D 102 (2020) 064003 [arXiv:2002.06801] [INSPIRE].

[15] ExHIC-P collaboration, Signatures of the vortical quark-gluon plasma in hadron yields, Phys. Rev. C 102 (2020) 021901 [arXiv:2002.10082] [INSPIRE].

[16] A.L. Watts et al., Colloquium: measuring the neutron star equation of state using x-ray timing, Rev. Mod. Phys. 88 (2016) 021001 [arXiv: 1602.01081] [INSPIRE]. 
[17] G. Policastro, D.T. Son and A.O. Starinets, The shear viscosity of strongly coupled $N=4$ supersymmetric Yang-Mills plasma, Phys. Rev. Lett. 87 (2001) 081601 [hep-th/0104066] [INSPIRE].

[18] R. Baier, P. Romatschke, D.T. Son, A.O. Starinets and M.A. Stephanov, Relativistic viscous hydrodynamics, conformal invariance, and holography, JHEP 04 (2008) 100 [arXiv: 0712.2451] [INSPIRE].

[19] S. Bhattacharyya, V.E. Hubeny, S. Minwalla and M. Rangamani, Nonlinear fluid dynamics from gravity, JHEP 02 (2008) 045 [arXiv:0712.2456] [INSPIRE].

[20] S. Bhattacharyya, S. Lahiri, R. Loganayagam and S. Minwalla, Large rotating AdS black holes from fluid mechanics, JHEP 09 (2008) 054 [arXiv:0708.1770] [INSPIRE].

[21] S. Bhattacharyya, R. Loganayagam, I. Mandal, S. Minwalla and A. Sharma, Conformal nonlinear fluid dynamics from gravity in arbitrary dimensions, JHEP 12 (2008) 116 [arXiv: 0809.4272] [INSPIRE].

[22] H. Bantilan, T. Ishii and P. Romatschke, Holographic heavy-ion collisions: analytic solutions with longitudinal flow, elliptic flow and vorticity, Phys. Lett. B 785 (2018) 201 [arXiv: 1803.10774] [INSPIRE].

[23] M. Garbiso and M. Kaminski, Hydrodynamics of simply spinning black holes \& hydrodynamics for spinning quantum fluids, JHEP 12 (2020) 112 [arXiv:2007.04345] [INSPIRE].

[24] S.W. Hawking, C.J. Hunter and M. Taylor, Rotation and the AdS/CFT correspondence, Phys. Rev. D 59 (1999) 064005 [hep-th/9811056] [INSPIRE].

[25] D.S. Berman and M.K. Parikh, Holography and rotating AdS black holes, Phys. Lett. B 463 (1999) 168 [hep-th/9907003] [INSPIRE].

[26] S.W. Hawking and H.S. Reall, Charged and rotating AdS black holes and their CFT duals, Phys. Rev. D 61 (2000) 024014 [hep-th/9908109] [INSPIRE].

[27] K. Landsteiner and E. Lopez, The thermodynamic potentials of Kerr-AdS black holes and their CFT duals, JHEP 12 (1999) 020 [hep-th/9911124] [INSPIRE].

[28] A.M. Awad and C.V. Johnson, Higher dimensional Kerr-AdS black holes and the AdS/CFT correspondence, Phys. Rev. D 63 (2001) 124023 [hep-th/0008211] [INSPIRE].

[29] G.W. Gibbons, M.J. Perry and C.N. Pope, The first law of thermodynamics for Kerr-anti-de Sitter black holes, Class. Quant. Grav. 22 (2005) 1503 [hep-th/0408217] [INSPIRE].

[30] B. Mcinnes, Applied holography of the AdS5-Kerr space-time, Int. J. Mod. Phys. A 34 (2019) 1950138 [arXiv: 1803.02528] [INSPIRE].

[31] J. Sonner, A rotating holographic superconductor, Phys. Rev. D 80 (2009) 084031 [arXiv: 0903.0627] [INSPIRE].

[32] A. Nata Atmaja and K. Schalm, Anisotropic drag force from $4 D$ Kerr-AdS black holes, JHEP 04 (2011) 070 [arXiv: 1012.3800] [INSPIRE].

[33] I. Aref'eva, A. Bagrov and A.S. Koshelev, Holographic thermalization from Kerr-AdS, JHEP 07 (2013) 170 [arXiv: 1305.3267] [INSPIRE]. 
[34] V. Cardoso, O.J.C. Dias, G.S. Hartnett, L. Lehner and J.E. Santos, Holographic thermalization, quasinormal modes and superradiance in Kerr-AdS, JHEP 04 (2014) 183 [arXiv: 1312.5323] [INSPIRE].

[35] J.B. Amado, B. Carneiro da Cunha and E. Pallante, On the Kerr-AdS/CFT correspondence, JHEP 08 (2017) 094 [arXiv:1702.01016] [InSPIRE].

[36] M. Cvetič, W.-J. Geng, H. Lü and C.N. Pope, BPS Kerr-AdS time machines, JHEP 07 (2018) 088 [arXiv:1801.08579] [INSPIRE].

[37] A. Castro, F. Larsen and I. Papadimitriou, $5 D$ rotating black holes and the $n A d S_{2} / n C F T_{1}$ correspondence, JHEP 10 (2018) 042 [arXiv: 1807.06988] [INSPIRE].

[38] S.S. Gubser, Drag force in AdS/CFT, Phys. Rev. D 74 (2006) 126005 [hep-th/0605182] [INSPIRE].

[39] C.P. Herzog, A. Karch, P. Kovtun, C. Kozcaz and L.G. Yaffe, Energy loss of a heavy quark moving through $N=4$ supersymmetric Yang-Mills plasma, JHEP 07 (2006) 013 [hep-th/0605158] [INSPIRE].

[40] A. Buchel, On jet quenching parameters in strongly coupled non-conformal gauge theories, Phys. Rev. D 74 (2006) 046006 [hep-th/0605178] [INSPIRE].

[41] E. Cáceres and A. Güijosa, Drag force in charged N=4 SYM plasma, JHEP 11 (2006) 077 [hep-th/0605235] [INSPIRE].

[42] J. Sadeghi and B. Pourhassan, Drag force of moving quark at the $N=2$ supergravity, JHEP 12 (2008) 026 [arXiv:0809.2668] [INSPIRE].

[43] S.-J. Sin and I. Zahed, Ampere's law and energy loss in AdS/CFT duality, Phys. Lett. B 648 (2007) 318 [hep-ph/0606049] [INSPIRE].

[44] T. Matsuo, D. Tomino and W.-Y. Wen, Drag force in SYM plasma with B field from AdS/CFT, JHEP 10 (2006) 055 [hep-th/0607178] [INSPIRE].

[45] K. Bitaghsir Fadafan, H. Liu, K. Rajagopal and U.A. Wiedemann, Stirring strongly coupled plasma, Eur. Phys. J. C 61 (2009) 553 [arXiv:0809. 2869] [inSPIRE].

[46] U. Gürsoy, E. Kiritsis, G. Michalogiorgakis and F. Nitti, Thermal transport and drag force in improved holographic QCD, JHEP 12 (2009) 056 [arXiv:0906.1890] [INSPIRE].

[47] M. Attems et al., Thermodynamics, transport and relaxation in non-conformal theories, JHEP 10 (2016) 155 [arXiv:1603.01254] [INSPIRE].

[48] M. Chernicoff, D. Fernandez, D. Mateos and D. Trancanelli, Drag force in a strongly coupled anisotropic plasma, JHEP 08 (2012) 100 [arXiv:1202.3696] [INSPIRE].

[49] D. Giataganas and H. Soltanpanahi, Heavy quark diffusion in strongly coupled anisotropic plasmas, JHEP 06 (2014) 047 [arXiv:1312.7474] [INSPIRE].

[50] I. Aref'eva, Holography for nonperturbative study of QFT, Phys. Part. Nucl. 51 (2020) 489 [INSPIRE].

[51] M. Lekaveckas and K. Rajagopal, Effects of fluid velocity gradients on heavy quark energy loss, JHEP 02 (2014) 068 [arXiv:1311.5577] [INSPIRE].

[52] K. Rajagopal and A.V. Sadofyev, Chiral drag force, JHEP 10 (2015) 018 [arXiv: 1505.07379] [INSPIRE]. 
[53] R. Rougemont, A. Ficnar, S. Finazzo and J. Noronha, Energy loss, equilibration, and thermodynamics of a baryon rich strongly coupled quark-gluon plasma, JHEP 04 (2016) 102 [arXiv: 1507.06556] [INSPIRE].

[54] D. Giataganas, Probing strongly coupled anisotropic plasma, JHEP 07 (2012) 031 [arXiv: 1202.4436] [INSPIRE].

[55] S. Chakraborty and N. Haque, Drag force in strongly coupled, anisotropic plasma at finite chemical potential, JHEP 12 (2014) 175 [arXiv:1410.7040] [INSPIRE].

[56] L. Cheng, X.-H. Ge and S.-Y. Wu, Drag force of anisotropic plasma at finite U(1) chemical potential, Eur. Phys. J. C 76 (2016) 256 [arXiv:1412.8433] [InSPIRE].

[57] E. Brehm, Heavy quarks in strongly coupled non-conformal plasmas with anisotropy, JHEP 06 (2019) 128 [arXiv:1711.08943] [InSPIRE].

[58] S.I. Finazzo, R. Critelli, R. Rougemont and J. Noronha, Momentum transport in strongly coupled anisotropic plasmas in the presence of strong magnetic fields, Phys. Rev. D 94 (2016) 054020 [Erratum ibid. 96 (2017) 019903] [arXiv: 1605.06061] [INSPIRE].

[59] K.A. Mamo, Energy loss of a nonaccelerating quark moving through a strongly coupled $N=4$ super Yang-Mills vacuum or plasma in strong magnetic field, Phys. Rev. D 94 (2016) 041901 [arXiv: 1606.01598] [INSPIRE].

[60] Z.-R. Zhu, S.-Q. Feng, Y.-F. Shi and Y. Zhong, Energy loss of heavy and light quarks in holographic magnetized background, Phys. Rev. D 99 (2019) 126001 [arXiv:1901.09304] [INSPIRE].

[61] Z.-Q. Zhang, Light quark energy loss in strongly coupled $N=4$ SYM plasma with magnetic field, Phys. Lett. B 793 (2019) 308 [INSPIRE].

[62] I.Y. Aref'eva, K. Rannu and P. Slepov, Energy loss in holographic anisotropic model for heavy quarks in external magnetic field, arXiv:2012.05758 [INSPIRE].

[63] O. Andreev, Drag force on heavy quarks and spatial string tension, Mod. Phys. Lett. A 33 (2018) 1850041 [arXiv:1707.05045] [INSPIRE].

[64] N. Drukker and S. Kawamoto, Circular loop operators in conformal field theories, Phys. Rev. D 74 (2006) 046002 [hep-th/0512150] [INSPIRE].

[65] R.C. Myers and M.J. Perry, Black holes in higher dimensional space-times, Ann. Phys. 172 (1986) 304.

[66] C. Erices and C. Martinez, Rotating hairy black holes in arbitrary dimensions, Phys. Rev. D 97 (2018) 024034 [arXiv: 1707.03483] [INSPIRE].

[67] D. Kubiznak and V.P. Frolov, Stationary strings and branes in the higher-dimensional Kerr-NUT-(A)dS spacetimes, JHEP 02 (2008) 007 [arXiv:0711.2300] [INSPIRE].

[68] M. Cvetič, H. Lü and C.N. Pope, Charged Kerr-de Sitter black holes in five dimensions, Phys. Lett. B 598 (2004) 273 [hep-th/0406196] [INSPIRE].

[69] I. Aref'eva, Theoretical studies of the formation and properties of quark-gluon matter under conditions of high baryon densities attainable at the NICA experimental complex, to be published in Phys. Part. Nucl.

[70] X.-G. Huang, P. Huovinen and X.-N. Wang, Quark polarization in a viscous quark-gluon plasma, Phys. Rev. C 84 (2011) 054910 [arXiv:1108.5649] [InSPIRE]. 
[71] L.P. Csernai, D.J. Wang, M. Bleicher and H. Stöcker, Vorticity in peripheral collisions at the facility for antiproton and ion research and at the JINR Nuclotron-based Ion Collider fAcility, Phys. Rev. C 90 (2014) 021904 [inSPIRE].

[72] M.I. Baznat, K.K. Gudima, A.S. Sorin and O.V. Teryaev, Femto-vortex sheets and hyperon polarization in heavy-ion collisions, Phys. Rev. C 93 (2016) 031902 [arXiv:1507.04652] [INSPIRE].

[73] D.E. Kharzeev, J. Liao, S.A. Voloshin and G. Wang, Chiral magnetic and vortical effects in high-energy nuclear collisions - a status report, Prog. Part. Nucl. Phys. 88 (2016) 1 [arXiv: 1511.04050] [INSPIRE].

[74] Y. Jiang, Z.-W. Lin and J. Liao, Rotating quark-gluon plasma in relativistic heavy ion collisions, Phys. Rev. C 94 (2016) 044910 [Erratum ibid. 95 (2017) 049904] [arXiv: 1602.06580] [INSPIRE].

[75] E.E. Kolomeitsev, V.D. Toneev and V. Voronyuk, Vorticity and hyperon polarization at energies available at JINR Nuclotron-based Ion Collider fAcility, Phys. Rev. C 97 (2018) 064902 [arXiv: 1801.07610] [INSPIRE].

[76] A. Sheykhi and S.H. Hendi, Charged rotating dilaton black branes in AdS universe, Gen. Rel. Grav. 42 (2010) 1571 [arXiv:0911.2831] [InSPIRE].

[77] M. Bravo Gaete, L. Guajardo and M. Hassaine, A Cardy-like formula for rotating black holes with planar horizon, JHEP 04 (2017) 092 [arXiv: 1702.02416] [INSPIRE].

[78] Z. Zhang, C. Shi, X. Luo and H.-S. Zong, Chiral phase transition in a rotating sphere, Phys. Rev. D 101 (2020) 074036 [arXiv:2003.03765] [InSPIRE].

[79] X. Chen, L. Zhang, D. Li, D. Hou and M. Huang, Gluodynamics and deconfinement phase transition under rotation from holography, arXiv:2010.14478 [INSPIRE].

[80] V. Toneev, O. Rogachevsky and V. Voronyuk, Evidence for creation of strong electromagnetic fields in relativistic heavy-ion collisions, Eur. Phys. J. A 52 (2016) 264 [arXiv: 1604.06231] [INSPIRE].

[81] A. Yamamoto and Y. Hirono, Lattice QCD in rotating frames, Phys. Rev. Lett. 111 (2013) 081601 [arXiv: 1303.6292] [INSPIRE].

[82] D. Kharzeev and A. Zhitnitsky, Charge separation induced by P-odd bubbles in QCD matter, Nucl. Phys. A 797 (2007) 67 [arXiv:0706.1026] [InSPIRE].

[83] D.T. Son and P. Surowka, Hydrodynamics with triangle anomalies, Phys. Rev. Lett. 103 (2009) 191601 [arXiv:0906 . 5044] [INSPIRE].

[84] D.E. Kharzeev and D.T. Son, Testing the chiral magnetic and chiral vortical effects in heavy ion collisions, Phys. Rev. Lett. 106 (2011) 062301 [arXiv: 1010.0038] [INSPIRE]. 